
\title{
25 Research Square \\ Effects of EHP-101 on inflammation and remyelination in murine models of Multiple Sclerosis
}

Carmen Navarrete

Emerald Pharmaceuticals

Adela García-Martin

Emerald Biotechnology España

Martín Garrido-Rodriguez

Universidad de Cordoba Facultad de Medicina y Enfermeria

Leyre Mestre

Cajal Institute, CSIC

Ana Feliu

Cajal Institute, CSIC

Carmen Guaza

Cajal Institute, CSIC

Marco A Calzado

Universidad de Cordoba Facultad de Medicina y Enfermeria

Eduardo Muñoz ( $\nabla$ fi1muble@uco.es)

Universidad de Cordoba Facultad de Medicina y Enfermeria https://orcid.org/0000-0001-8478-5842

\section{Research}

Keywords: Multiple Sclerosis, EHP-101, Transcriptomic, Inflammation, Remyelination

Posted Date: January 23rd, 2020

DOI: https://doi.org/10.21203/rs.2.21717/v1

License: (1) (1) This work is licensed under a Creative Commons Attribution 4.0 International License.

Read Full License

Version of Record: A version of this preprint was published at Neurobiology of Disease on September 1st, 2020. See the published version at https://doi.org/10.1016/j.nbd.2020.104994. 


\section{Abstract}

Background: Multiple Sclerosis (MS) is characterized by a combination of inflammatory and neurodegenerative processes in the spinal cord and the brain. Natural and synthetic cannabinoids such as VCE-004.8 have been studied in preclinical models of MS and represent promising candidates for drug development. VCE-004.8 is a multitarget synthetic cannabidiol (CBD) derivative acting as a dual Peroxisome proliferator-activated receptor-gamma/ Cannabinoid receptor type 2 (PPAR y /CB 2) ligand agonist that also activates the Hypoxia-inducible factor (HIF) pathway. EHP-101 is an oral lipidic formulation of VCE-004.8 that has shown efficacy in several preclinical models of autoimmune, inflammatory, fibrotic and neurodegenerative diseases. Methods: The efficacy of EHP-101 in vivo was evaluated in two murine models of MS, the experimental autoimmune encephalomyelitis (EAE) and cuprizone-induced demyelination models. In EAE, transcriptomic analysis was performed by RNA-Seq and qPCR, and inflammatory and myelination markers were detected by immunohistochemistry (IHC) and confocal microscopy in both models of MS. Results: EHP-101 alleviated clinical symptomatology in EAE and transcriptomic analysis demonstrated that EHP-101 prevented the expression of many inflammatory genes closely associated with MS pathophysiology in the spinal cord. EHP-101 normalized the expression of several genes associated with oligodendrocyte function such as Teneurin 4 (Tenm4) and Gap junction gamma-3 (Gjc3) that were downregulated in EAE. EHP-101 treatment prevented microglia activation and demyelination in both the spinal cord and the brain. Moreover, EAE was associated with a loss in the expression of Oligodendrocyte transcription factor 2 (Olig2) in the corpus callosum, a marker for oligodendrocyte differentiation, which was restored by EHP-101 treatment. In addition, EHP-101 enhanced the expression of glutathione S-transferase pi (GSTpi), a marker for mature oligodendrocytes in the brain. We also found that a diet containing $0.2 \%$ cuprizone for six weeks induced a clear loss of myelin in the brain measured by Cryomyelin staining and Myelin basic protein (MBP) expression. Moreover, EHP-101 also prevented cuprizone-induced microglial activation, astrogliosis and reduced axonal damage. Conclusions: Our results provide evidence that EHP-101 showed potent antiinflammatory activity, prevented demyelination and enhanced remyelination. Therefore, EHP-101 represents a promising drug candidate for the potential treatment of different forms of MS.

\section{Background}

Multiple sclerosis (MS) is an autoimmune disease that affects the central nervous system (CNS) and is characterized by pathological changes, including neuroinflammation, demyelination and axon injury [1-3]. The spontaneous repair of damaged myelin sheaths and axons has been described during the remission period of classical relapsing-remitting MS (RRMS), where demyelinated axons could be rewrapped by the regenerated myelin sheath, thus ameliorating axonal dysfunction. In this sense, the remission period is also considered the period of remyelination $[4,5]$, which is important because it could be a key time for the treatment of RRMS patients with drugs preventing inflammation and enhancing remyelination.

Small molecules including cannabinoids acting at druggable targets of the endocannabinoid system (ECS) are being explored for the management of CNS pathologies including MS [6]. In this sense, several 
lines of evidence suggested a role for the ECS in oligodendrocyte function and remyelination activity in MS [7-9]. The ECS is composed by the G-protein coupled receptors Cannabinoid type $1\left(\mathrm{CB}_{1}\right)$ and type 2 $\left(\mathrm{CB}_{2}\right)$, endocannabinoids and the enzymes regulating their synthesis and catabolism. In addition, cannabinoids of a different nature also target ionotropic receptors of the Transient receptor potential channels (TRP channels) family and nuclear receptors such as peroxisome proliferator-activated receptors (PPARs) $[10,11]$. $\mathrm{CB}_{1}$ receptors are expressed mainly in the CNS at neuronal terminals and regulate neurotransmitter release and psychoactive processes. In contrast, $\mathrm{CB}_{2}$ receptors are located primarily in the peripheral immune system, and its expression is increased during neuroinflammation on activated microglia in the CNS $[12,13]$. Key considerations for developing $\mathrm{CB}_{2}$ receptor agonists include absence of psychoactive effects, sustained anti-inflammatory activity, tissue/cell protection, lack of cardiovascular adverse effects and efficacy in several disease models on neuroinflammation including MS [12, 14-17].

PPARs are members of the nuclear hormone receptor superfamily of ligand-activated transcriptional factors [18] with well-identified regulatory roles in lipid and glucose homeostasis and adipocyte differentiation [19]. In addition to adipocytes and hepatocytes, PPARg has been shown to be expressed in different CNS cells and in immune cells [20]. Furthermore, PPARg has been described as an important factor in the regulation of the immune response [21]. In this sense, PPARg activation has been shown to suppress the expression of inflammatory cytokines in astrocytes and macrophages/microglia [22-24]. Furthermore, PPARg stimulated oligodendrocyte differentiation from neural stem cells [25], promoted and accelerated the differentiation of oligodendrocyte progenitor cells in vitro with an additional increase in antioxidant defences $[26,27]$ and increased lipid production and terminal differentiation of cultured oligodendrocytes [28], thus suggesting an additional possible protective role of PPARg in MS as a mediator of remyelination. The neuroprotective effects of PPARs, including PPARg, have also been widely documented in vitro in various experimental paradigms of neurodegeneration, broadening its potential therapeutic perspectives in MS [29-32].

Although most current therapies for MS are directed towards modulation of the exacerbated immune response [33], novel therapies aimed at axonal remyelination are urgently needed. A novel approach to achieve this would be the hypoxia preconditioning process which, induced by mild oxygen depletion, is beneficial in a wide number of neurological disorders, including MS [34,35]. The cellular adaptation to severe or mild hypoxia is very fast and involves the activation of the hypoxia-inducible factor (HIF)- $1 \mathrm{a}$, whose activation may play a role in the inflammatory and the remitting phases of MS (reviewed by [36]). In addition, there is evidence suggesting that activation of the HIF pathway may also be linked to neuroprotection and perhaps remyelination [37]. For instance, erythropoietin (EPO), whose gene is dependent on HIF activation, is neuroprotective in different animal models of MS [38, 39].

We have previously shown that VCE-004.8 is a promising cannabidiol (CBD) derivative acting as a dual agonist of PPARg and $\mathrm{CB}_{2}$ that also activates the HIF pathway [17]. Indeed, VCE-004.8 prevented neuroinflammation and demyelination in two different murine models of MS, namely EAE and Theiler's 
virus-induced demyelinating disease [17]. EHP-101 is an oral formulation of VCE-004.8 that also showed efficacy in a murine model of systemic sclerosis (SSc) [40, 41]. EHP-101 has completed a Phase I clinical study (clinicaltrial.gov: NCT03745001) and initiation of Phase II studies in SSc and MS patients are being planned. Herein we show the efficacy of EHP-101 in preventing neuroinflammation and demyelination in EAE and enhancing remyelination in the cuprizone model of demyelination.

\section{Methods}

Compounds. EHP-101 is a lipidic formulation of VCE-004.8 [(1'R,6'R)-3-(Benzylamine)-6-hydroxy-3'methyl-4-pentyl-6'-(prop-1-en-2-yl) [1,1'bi(cyclohexane)]-2',3,6-triene-2,5-dione)] [41]. The chromatographic purity of VCE-004.8 in EHP-101 was 97.6\%.

Animals. All experiments were performed in strict accordance with European Union (EU) and governmental regulations. Handling of animals was performed in compliance with the guidelines of animal care set by the EU guidelines 86/609/EEC, and the Ethics Committees on Animal Experimentation at the Cajal Institute (CSIC, Madrid) and the University of Córdoba (UCO, Córdoba, Spain) approved all the procedures described in this study (for EAE at Cajal Institute protocol number: 96 2013/03 CEEA-IC and for cuprizone model at UCO protocol number: 2018PI/02 (UCO). Measures to improve welfare assistance and clinical status as well as endpoint criteria were established to minimize suffering and ensure animal welfare. Briefly, wet food pellets are placed on the bed-cage when the animals begin to develop clinical signs to facilitate access to food and hydration. For the EAE model, female C57BL/ 6 mice were purchased from Harlan (Barcelona, Spain) and for the cuprizone model, male C56BL/ 6 mice were purchased from Janvier Labs (Le Genest-Saint-Isle, France). All animals were housed in the animal facilities under the following controlled conditions: $12 \mathrm{~h}$ light/dark cycle; temperature $20^{\circ} \mathrm{C}\left( \pm 2^{\circ} \mathrm{C}\right)$ and 40 $50 \%$ relative humidity with free access to standard food and water.

Induction and assessment of EAE. EAE was induced in C57BL/ 6 female mice at 6-8 weeks of age by subcutaneous immunization with Myelin Oligodendrocyte Glycoprotein Peptide Fragment 35-55 (MOG35-55) (300 $\mu$ g: peptide synthesis section, CBM, CSIC, Madrid, Spain) and $200 \mu \mathrm{g}$ of Mycobacterium tuberculosis (H37Ra Difco, Franklin Lakes, NJ, USA) in a 1:1 mix with incomplete Freund's adjuvant (CFA, Sigma). On the same day and 2 days later, mice were injected intraperitoneally with $200 \mathrm{ng}$ of pertussis toxin (Sigma) in $0.1 \mathrm{ml}$ Phosphate buffer saline (PBS). Control animals (CFA) were inoculated with the same emulsion without MOG and they did not receive pertussis toxin. Treatment started at day 8 post-immunization when animals showed the first symptoms of the disease and consisted of daily oral EHP-101 administration $(1,5,10$ and $20 \mathrm{mg} / \mathrm{kg})$ for the following 21 days. The mice were examined daily for clinical signs of EAE and disease scores were measured as follows: 0 , no disease; 1, limb tail; 2 , limb tail and hind limb weakness; 3 , hind limb paralysis; 4 , hind limb and front limb paralysis; 5 , moribund and death. All animals were sacrificed at 28 days for further analysis. 
Cuprizone-induced demyelination. To induce demyelination, 8-week old C57BL/6 male mice were fed with $0.2 \%$ cuprizone (CPZ) TD. 140800 diet (Envigo, Barcelona, Spain) for six weeks. Control group (no demyelination) was fed with control mouse TD.00217 diet (Envigo, Barcelona, Spain) for the entire period. To study the effect on remyelination, EHP-101 was administered once daily by oral gavage at $20 \mathrm{mg} / \mathrm{kg}$ from week six. For comparison, animals in the CPZ control group (maximal demyelination) received the same volume of vehicle by gavage. To study the dynamic effect of EHP-101 on remyelination, animals in each group were sacrificed at weeks $6,7(6+1 \mathrm{~W}), 8(6+2 \mathrm{~W})$ for further analysis.

Tissue processing. Mice were anesthetized by i.p. administration of a ketamine-xylazine or pentobarbital solution and they were transcardially perfused with saline $0.9 \%$. The spinal cord was obtained by extrusion with saline. Brain and cervical spinal cord were immediately frozen and kept at $-80^{\circ} \mathrm{C}$ for RTPCR analysis. The remaining brain and spinal cord were fixed in $4 \%$ paraformaldehyde in $0.1 \mathrm{M} \mathrm{PBS}$, washed in $0.1 \mathrm{M}$ PBS, cryoprotected with a $15 \%$ and then a $30 \%$ solution of sucrose in $0.1 \mathrm{M}$ PBS, and frozen at $-80^{\circ} \mathrm{C}$. Free-floating brain and thoracic spinal cord sections $(50 \mu \mathrm{m}$ thick: Leica Microsystems CM1900 cryostat, Barcelona, Spain) were then processed for immunohistochemistry or immunofluorescence. In the case of cuprizone model whole brains were fixed, cryoprotected and frozen at $-80^{\circ} \mathrm{C}$ for further analysis.

Immunohistochemistry analysis. For IHC analysis, free-floating thoracic spinal cord (50 $\mu \mathrm{m})$ sections were washed with $0.1 \mathrm{M}$ Phosphate buffer $(\mathrm{PB})$. Endogenous peroxidase activity was inhibited with $3.3 \%$ hydrogen peroxide in methanol. The sections were blocked with $2.5 \%$ normal horse serum and then incubated overnight at $4^{\circ} \mathrm{C}$ in blocking buffer with a rabbit anti-Teneurin 4 antibody (1:50: Novus Biological, Colorado, USA). Slides were incubated with ImmPRESS reagent (Vector Laboratories; Burlingame, Ca, USA) and then developed with diaminobenzidine chromogen (Merck, Darmstadt, Germany). Samples were photographed, digitalized using a Leica DFC420c camera and analyzed using Image $\mathrm{J}$ software. Myelin integrity was analyzed using the Hito CryoMyelinStain ${ }^{\text {TM }}$ Kit (Gold phosphate complex Myelin Staining Kit) following manufacturer's recommendation (Hitobiotech Corp., Kingsport, TN, USA).

Confocal microscopy analysis. For antigen retrieval, spinal cord or brain sections were boiled for $10 \mathrm{~min}$ in sodium citrate buffer (10 mM, pH 6.0) or Tris-EDTA buffer (10 mM Tris Base, $1 \mathrm{mM}$ EDTA, $0.05 \%$ Tween 20, pH 9.0) (Sigma-Aldrich, St. Louis, MO, USA). The sections were washed three times in PBS. Nonspecific antibody-binding sites were blocked for $1 \mathrm{~h}$ at room temperature with $3 \%$ bovine serum albumin (BSA) (Sigma-Aldrich, St. Louis, MO, USA in PBS). Next, the sections were incubated overnight at 
$4{ }^{\circ} \mathrm{C}$ with the following primary antibodies diluted in PBS with $3 \%$ BSA: microglia cells were stained with a rabbit anti-ionized calcium binding adaptor molecule 1 (Iba-1) antibody $(1: 1,000$; Wako Chemical Pure Industry, Osaka, Japan), astrocytes were stained with a mouse anti-glial fibrillary acidic protein (GFAP) antibody (1:500, Santa Cruz Biotechnology, Santa Cruz, CA, USA), myelin basic protein was marked with a rabbit anti-Myelin Basic Protein (MBP) antibody (1:1000; Abcam, Cambridge, UK), oligodendrocytes were marked with a mouse anti-Olig2 (1:100, Santa Cruz, CA, USA) and a rabbit anti-GSTPi (1:250, Abcam, Cambridge, UK) axonal damage was determined with a mouse anti-Neurofilament $\mathrm{H}(\mathrm{NF}-\mathrm{H})$ Nonphosphorylated antibody (SMI-32) (1:50; Biolegend, CA, USA). After extensive washing in PBS, slides were incubated with secondary antibodies for $1 \mathrm{~h}$ at room temperature in the dark. The immunoreactions were revealed using anti-rabbit Texas Red (1:100), anti-mouse/rabbit Alexa 488 (1:100) obtained from Thermo Fischer Scientific, Walthamm, MA, USA. The slides were then mounted using Vectashield Antifade Mounting Medium with DAPI (Vector Laboratories, Burlingame, $\mathrm{Ca}$, USA). All images were acquired using a spectral confocal laser-scanning microscope LSM710, (Zeiss, Jena, Germany) with a 20×/0.8 Plan-Apochromat lens and quantified in 9-15 randomly chosen fields using Image J software (http://rsbweb.nih.gov/ij/).

RNA-Seq and bioinformatic analysis. Total RNA was isolated from spinal cord tissue using QIAzol lysis reagent (Qiagen, Hilden, Germany) and purified with RNeasy Lipid Tissue Mini kit (Qiagen). Then, samples were processed for high throughput sequencing using poly-A selection with the TruSeq Stranded mRNA Library Prep Kit (Cat. No. RS-122-2101, Illumina, San Diego, CA, USA). In brief, $1 \mu \mathrm{g}$ of total RNA from each sample was used to construct a cDNA library, followed by sequencing on the lllumina HiSeq 2500 system with single end 50 bp reads and $\sim 40$ millions of reads per sample ( $n=3$ per group). FASTQ files were pre-processed with Trimmomatic (v0.36) [42] and aligned to mouse genome assembly mm10 using HISAT2 (v2.1.0) [43]. Then, counts per gene matrix were obtained with featureCounts (v1.6.1) [44] using the in-built RefSeq annotation for $\mathrm{mm} 10$ genome assembly and the differential expression analysis was carried out using DESeq2 (v1.20.0) [45], excluding genes with less than 15 counts across all samples. The functional over-representation analyses were performed using EnrichR [46] and clusterProfiler [47]. All the $P$ values were adjusted to control the false discovery rate (FDR) using the Benjamini and Hochberg approach [48]. RNA-seq data have been deposited in the Gene Expression Omnibus databank (accession no. GSE131854).

Quantitative reverse transcriptase-PCR. Total RNA $(1 \mu \mathrm{g})$ was retrotranscribed using the iScript cDNA Synthesis Kit (Bio-Rad, Hercules, CA, USA) and the CDNA analyzed by real-time PCR using the iQTM SYBR Green Supermix (Bio-Rad) and a CFX96 Real-time PCR Detection System (Bio-Rad). Glyceraldehyde-3Phosphate Dehydrogenase (GAPDH) gene was used to standardize mRNA expression in each sample. Gene expression was quantified using the 2- $\Delta \Delta \mathrm{Ct}$ method and the percentage of relative expression against controls was represented. The primers used in this study are described in Table 1. 
Data analysis. All the in vivo data are expressed as the mean \pm SEM. One-way analysis of variance (ANOVA) followed by the Tukey's post-hoc test for parametric analysis or Kruskal-Wallis post-hoc test in the case of non-parametric analysis tests were used to determine the statistical significance. The level of significance was set at $\mathrm{p} \otimes 0.05$. Statistical analyses were performed using GraphPad Prism version 8.00 (GraphPad, San Diego, CA, USA).

\section{Results}

\section{EHP-101 attenuates clinical severity and neuroinflammation in EAE.}

The efficacy of EHP-101 in MS was first evaluated in EAE, performing the treatments at an early stage of the disease since mice received increasing doses of EHP-101 at day 8 post-immunization (p.i.). Subcutaneous immunization with MOG35-55 induced EAE in all mice that received the vehicle alone. All vehicle-treated mice developed a disease that peaked by day 16 p.i. and maintained at day 28 p.i. By contrast, the reduced clinical score showed therapeutic efficacy of EHP-101 with all the dose levels tested, with the highest dose $(20 \mathrm{mg} / \mathrm{kg}$ ) able to prevent the symptoms completely (Figure 1a $\mathrm{p}=0.0002$ $E A E+E H P-10120 \mathrm{mg} / \mathrm{kg}$ vs EAE+Vehicle; $p=0.0046 \mathrm{EAE}+\mathrm{EHP}-10110 \mathrm{mg} / \mathrm{kg}$ vs EAE+Vehicle; $p=0.0068$ $E A E+E H P-1015 \mathrm{mg} / \mathrm{kg}$ vs EAE+Vehicle). Clinical score data from Figure 1 used to determine the area under curve as shown in Figure $1 b$ ( $p \otimes 0.0001$ EAE+EHP-101 1/5/10/20 mg/kg vs EAE+Vehicle) clearly demonstrated that EHP-101 improved symptomatology in a dose-dependent manner.

To determine whether EHP-101 was able to target neuroinflammation in EAE, microgliosis and astrogliosis were evaluated in the spinal cord. Histopathological analysis showed that the extensive

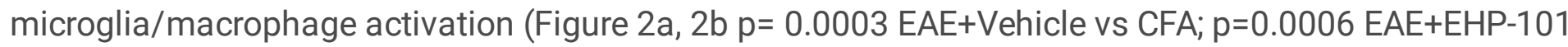

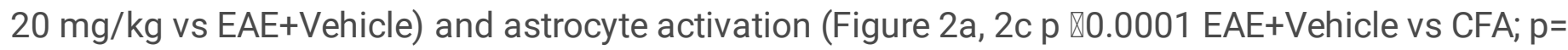
$0.0051 \mathrm{EAE}+\mathrm{EHP}-10120 \mathrm{mg} / \mathrm{kg}$ vs EAE+Vehicle) in the spinal cord of EAE mice evidenced by both Iba-1 and GFAP staining was greatly reduced by EHP-101.

MS pathology is characterized by focal demyelinating lesions in the CNS at both spinal cord and brain levels. Therefore, to determine the extent of demyelination, myelin was evaluated by MBP immunolabelling. A clear demyelination was found in the spinal cord of EAE mice that was significantly prevented by EHP-101 treatment (Figure 2a, 2d p=0.0001 EAE+Vehicle vs CFA; $p$ 『0.0001 EAE+EHP-101 vs $\mathrm{EAE}+\mathrm{Vehicle})$.

Cerebral cortical demyelination as well as callosal pathology are widely recognized features of MS [4951]. In addition, the cerebral cortex plays a central role in interhemispheric communication, and callosal atrophy in MS patients has been shown to correlate with disability status [52-54]. Therefore, we also examined whether these structures might also be affected in EAE mice. An increase in inflammatory lesions was seen throughout the EAE forebrain (Figure 3a). Specifically, we observed that microglial reactivity was increased in corpus callosum of EAE mice and the treatment with EHP-101 reverted the 
microgliosis process (Figure $3 b p=0.0002$ EAE+Vehicle vs CFA; $p=0.0395$ EAE+EHP-101 $20 \mathrm{mg} / \mathrm{kg}$ vs $\mathrm{EAE}+$ Vehicle). Furthermore, brain sections from EAE-affected mice were also analyzed for the distribution of MBP reactivity. MBP immunoreactivity appeared significantly reduced in cerebral cortex (Figure $3 c p=$ $0.0159 \mathrm{EAE}+$ Vehicle vs CFA; $\mathrm{p}=0.0024 \mathrm{EAE}+\mathrm{EHP}-10120 \mathrm{mg} / \mathrm{kg}$ vs $\mathrm{EAE}+\mathrm{Vehicle}$ ) and this loss of myelin expression was strongly reverted by EHP-101 treatment. Moreover, EAE is associated with a loss in the expression of Olig2 in the corpus callosum, a marker for the onset of oligodendrocyte differentiation, which was restored by EHP-101 treatment (Figure 3d $p \otimes 0.0001 \mathrm{EAE}+$ Vehicle vs CFA; $p=0.0008 \mathrm{EAE}+\mathrm{EHP}$ $10120 \mathrm{mg} / \mathrm{kg}$ vs EAE+Vehicle). In addition, EHP-101 enhanced the expression of glutathione Stransferase pi (GSTpi), a cytosolic isoenzyme used as a marker for mature oligodendrocytes in the brain (Figure $3 e \mathrm{p}=0.0222 \mathrm{EAE}+\mathrm{EHP}-10120 \mathrm{mg}$ vs EAE+Vehicle). These data are indicative of the potential of EHP-101 to prevent demyelination in an MS murine model.

\section{EHP-101 normalizes EAE transcriptomic signature in the spinal cord}

To evaluate the global expression changes produced by EHP-101 treatment, we performed an RNA-Seq analysis of the spinal cords from mice in the following conditions: Control, EAE and EAE with EHP-101 treatment $(20 \mathrm{mg} / \mathrm{kg})$. Sequencing data for three biological replicates were obtained for each experimental group. Then, we compared the transcriptomic profile between the different conditions to get a first insight into the changes occurring in the model, with or without treatment. As expected, we found many changes, both in magnitude and significance in EAE mice compared to the group treated with EHP101 (Figure 4a). Then, to evaluate those changes at a biological level, we performed an overrepresentation analysis using genes that surpassed the cutoff of an adjusted $\mathrm{P}<0.05$ and absolute fold change $>2$ in the EAE vs control and EAE+EHP-101 vs EAE comparisons. The more significant enrichments were found in the groups of upregulated genes by EAE and downregulated genes by the treatment. We observed a complementary signature between those two groups, where terms like "neutrophil-mediated immunity", "inflammatory response" or "cytokine-mediated signaling pathway" appeared, highlighting an anti-inflammatory effect of the EHP-101 treatment on the spinal cord (Figure 4b). The heatmap in Figure 4c represents genes from the "cytokine-mediated signaling pathway" that are induced by EAE and downregulated by EHP-101. Furthermore, to confirm this anti-inflammatory effect of EHP-101 in the spinal cord we determined the gene expression by RT-PCR of several genes such as Interleukin 6 (II6), Tissue inhibitor matrix metalloproteinase 1 (Timp1), vascular cell adhesion molecule 1 (Vcam), Interleukin 1 beta (II1 b), C-C Motif Chemokine Ligand 4 (Ccl4) and C-C Motif Chemokine Ligand 2 (Ccl2). We show in Figure 4d that EHP-101 treatment downregulated the expression of these genes upregulated in EAE mice (II6: $p=0.0360 \mathrm{EAE}+\mathrm{Vehicle} \mathrm{vs} \mathrm{CFA;} p=0.0451 \mathrm{EAE}+\mathrm{EHP}-10120 \mathrm{mg} / \mathrm{kg}$ vs EAE+Vehicle; Timp1: $p=$ =0.0001 EAE+Vehicle vs CFA; $p=0.0001 \mathrm{EAE}+\mathrm{EHP}-10120 \mathrm{mg} / \mathrm{kg}$ vs EAE+Vehicle; VCAM: $p=0.0058 E A E+V e h i c l e ~ v s ~ C F A, ~ p=0.0381 E A E+E H P-10120 \mathrm{mg} / \mathrm{kg}$ vs EAE+Vehicle; IL1 $\mathrm{b}: \mathrm{p}=$

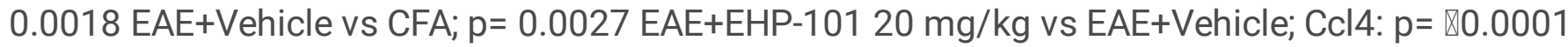

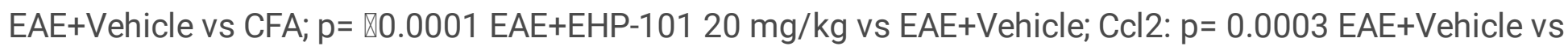
$C F A ; p=0.0054 E A E+E H P-101$ vs EAE+Vehicle), thus validating the results found in the RNA-Seq analysis. 
Next, we performed a second analysis to explore changes in the opposite direction to the pattern shown by the pro-inflammatory genes. Thus, we selected down-regulated genes in the EAE vs control comparison and up-regulated in EAE+EHP-101 vs EAE comparison. We intersected both groups of genes to evaluate the overlap between them, resulting in a total of 193 genes downregulated in the untreated model that increased their expression in response to the treatment (Figure 5a). Then we performed a second functional analysis, using the list of overlapping genes as input, to explore the most significantly enriched Gene Ontology (GO) terms. As depicted in Figure 5b, we found several terms related to the metabolic process of sterols and hydroxy compounds at the top of the list. However, given the background of the disease, we decided to focus on the "myelination" process. To explore the changes of features belonging to this annotation, we depicted the expression levels of genes that produced this result in the heatmap shown in Figure 5c. This allowed us to identify several key genes of the myelination process that were restoring their levels with EHP-101 treatment. Interestingly, these results indicated that EHP-101 normalized the expression of several genes associated with oligodendrocyte function, such as Gap junction gamma-3 (Gjc3), also called Connexin 29, and Teneurin-4 (Tenm4) that were downregulated in EAE. These results are relevant since Tenm4 has been described as a critical regulator of oligodendrocyte differentiation and CNS myelination [55]. To validate the transcriptomic analysis, we studied the expression of Gjc3 and Tenm4 by RT-PCR (Figure 5d Tenm4: $p=0.0020$ EAE+Vehicle vs CFA; $p=0.0032 \mathrm{EAE}+\mathrm{EHP}-10120 \mathrm{mg} / \mathrm{kg}$ vs EAE+Vehicle; Gjc3: $p=0.0006 \mathrm{EAE}+\mathrm{Vehicle}$ vs CFA; $p=0.0462$ $\mathrm{EAE}+\mathrm{EHP}-10120 \mathrm{mg} / \mathrm{kg}$ vs EAE+Vehicle) and the protein levels by IHC. As depicted in Figure $5 \mathrm{e}(\mathrm{p}=\mathbb{\nabla}$

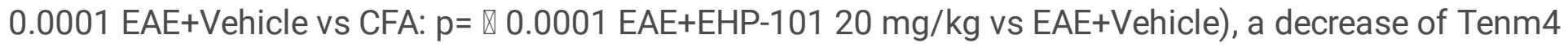
expression was observed in white matter of spinal cord compared to the CFA group which was prevented by EHP-101 treatment. Taken together, these results are indicative of the potential of EHP-101 to prevent demyelination in EAE model.

\section{EHP-101 accelerates remyelination in cuprizone-challenged mice.}

To evaluate the effect of EHP-101 on remyelination during the acute CPZ-induced demyelination protocol (Figure 6a), brain coronal sections from animals after 6 weeks of CPZ 0.2\% diet and 2 weeks of EHP-101 treatment were evaluated. In this model, EHP-101 treatment was started after removal of the CPZ diet to study the effect of EHP-101 on spontaneous remyelination. First, the evaluation of MBP was determined by CryoMyelin and IHC staining (Figure $6 \mathrm{~b}$ and $6 \mathrm{c}$, respectively). Spontaneous recovery from demyelination was insignificant after 1 and 2 weeks in untreated mice but remyelination was significantly accelerated by EHP-101 treatment in both the corpus callosum (Figure $6 \mathrm{~d} p=\otimes 0.0001 \mathrm{CPZ6W}, \mathrm{CPZ} 6+1 \mathrm{~W}$,

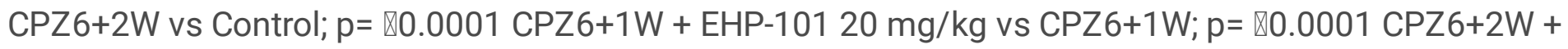
EHP-101 $20 \mathrm{mg} / \mathrm{kg}$ vs CPZ6+2W) and the cerebral cortex (Figure 6e $p=\rrbracket 0.0001 \mathrm{CPZ6W}, \mathrm{CPZ6}+1 \mathrm{~W}$,

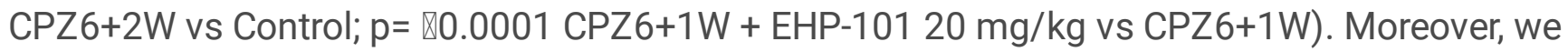
investigated the effect of EHP-101 on neuroinflammation-associated glial activation by staining Iba- $1^{+}$ and $\mathrm{GFAP}^{+}$cells in the corpus callosum. In control mice, low expression levels of $\mathrm{Iba}^{-}{ }^{+}$and $\mathrm{GFAP}^{+}$cells were detected but mice exposed to CPZ showed microglial and astrocytic activation, which was 
attenuated by EHP-101 treatment (Figure 7a and 7b). Quantitative assessment also showed a significant increase in the number of $\mathrm{Iba}^{+}$and $\mathrm{GFAP}^{+}$cells in corpus callosum upon $\mathrm{CPZ}$ intoxication. Microgliosis and astrocytic activation was ameliorated after 1 week of EHP-101 treatment (Figure $7 c p=\unrhd 0.0001$ CPZ6W, CPZ6+1W, CPZ6+2W vs Control; $p=0.0017$ CPZ6+1W + EHP-101 20 mg/kg vs CPZ6+1W; Figure $7 d p=\otimes 0.0001 \mathrm{CPZ6W}, C P Z 6+1 \mathrm{~W}$ vs Control; $p=0.0017 \mathrm{CPZ6}+2 \mathrm{~W}$ vs Control). To examine the effects of EHP-101 on cuprizone-induced demyelination of axons in the corpus callosum, we investigated the nonphosphorylated form of neurofilament proteins (SMI-32 staining). Although SMI-32 immunoreactivity is normally seen in axons, its accumulation in axonal spheroids is a characteristic of axonal pathology. Increased SMI-32 labeling after 6 and 7 weeks of CPZ intoxication demonstrated that there was a significant effect on axons and this effect was ameliorated after 1 week of EHP-101 treatment (Figure 8).

\section{Discussion}

Natural products, including phytocannabinoids, have been successfully used for the development of synthetic and semisynthetic derivatives with improved bioactivities [56]. We have developed the compound VCE-004.8, a synthetic derivative of CBD, which is a dual agonist for PPARg/ $\mathrm{CB}_{2}$ that also inhibits the activity of HIF prolyl hydroxylases (PHDs) [17, 57]. Therefore, VCE-004.8 is targeting several pathways that may have a positive effect on neuroinflammation and remyelination as observed in EAE and Theiler's Murine Encephalomyelitis Virus-induced demyelinating models [17]. Herein we have studied the effect of EHP-101, an oral lipidic formulation of VCE-004.8, in two of the most commonly used models of demyelination, which are EAE and toxically induced demyelination via cuprizone [58].

$\mathrm{EAE}$ in $\mathrm{C} 57 \mathrm{BI} / 6$ mice has generally been thought to predominantly target the spinal cord, leading to sensory and motor impairments. Nevertheless, it is also recognized that EAE involves other CNS structures including the cerebellum and the hippocampus $[59,60]$. Our data clearly indicate that EHP-101 is effective in alleviating neuroinflammation in the spinal cord, in the cerebral cortex and in the corpus callosum. In the EAE model we cannot distinguish whether the effect of EHP-101 occurs in the peripheral immune system, in the CNS or both. It has been demonstrated that the blood-brain-barrier (BBB) is disrupted in EAE allowing for the migration of autoimmune cells and molecules to the brain [61]. However, it is likely that EHP-101 may exert anti-inflammatory effects by acting both in the peripheral immune system and in the CNS. For instance, EHP-101 showed anti-inflammatory activity in another autoimmune disease, systemic sclerosis, where the BBB is not affected [41] and herein we show that EHP-101 also alleviates neuroinflammation in CPZ-intoxicated mice. CPZ-induced demyelinating lesions are characterized by severe oligodendrocyte loss and demyelination with concomitant activation of microglia and astrocytes, but it does not induce BBB damage [62] and lacks the characteristic T-cell infiltration and consequently the peripheral autoimmune component of the disease [2].

The mechanism of action of EHP-101 in the remyelination process is still unknown but it can be probably related to the HIF pathway [17]. Extensive experimental studies have revealed that activating HIF-1a by inhibiting the activation of PHDs can provide neuroprotection and perhaps remyelination mainly from the increased expression of HIF-1 target genes, which combat oxidative stress, improve blood oxygen and 
glucose supply, promote glucose metabolism, regulate iron homeostasis and block cell death signal pathways. Increasing HIF-1 activity may be an important potential strategy to prevent the onset or to ameliorate the pathogenesis of neurodegenerative diseases [63]. Interestingly, the improvement of the myelination index was paralleled by enhancement of oligodendrocyte progenitor cell (OPC) proliferation, platelet-derived growth factor (PDGF)a-receptor expression, and precursor migration from the CC midline to the lateral parts followed by an induction of the expression of myelin protein. In addition, early astrogliosis in the demyelinated areas paralleled a moderate stimulation of insulin-like growth factor (IGF)-1 expression [2]. IGF-1 synergizes with fibroblast growth factor (FGF)-2 to stimulate oligodendrocyte progenitor entry into the cell cycle [64]. This is of particular interest because IGF-1 induced HIF-1 activation that can be mimicked by VCE-004.8 in the brain, and PDGFa and FGF2 are also regulated by VCE-004.8-mediated activation of the HIF pathway $[17,65,66]$.

Demyelination and partial axonal damage in MS lesions are closely associated with reactive activation of microglial cells which are seen in close contact with axons, that reveal acute axonal injury, such as the formation of axonal spheroids or a disturbance of fast axonal transport $[67,68]$. Reactive microglia produce a large array of toxic and proinflammatory molecules, which triggers myelin destruction, oligodendrocyte deterioration, axon damage and even neuronal loss [69] [70]. Here we found that oral EHP-101 also prevented microglia activation and demyelination in both spinal cord and brain suggesting that VCE-004.8 penetrates the brain in EAE mice after oral absorption. Moreover, we also found that EHP101 preserves the axonal structure ameliorating the typical accumulation of spheroids of SMI-32 used as a marker of axonal damage in CPZ intoxicated mice [67, 71, 72]. Again, this result suggests that VCE004.8 can also cross the BBB, which is not affected in the CPZ model [62].

Oligodendrocyte progenitor cells (OPCs) are produced from neuroepithelial stem cells and subsequently proliferate and migrate throughout the entire spinal cord [73]. During differentiation, oligodendrocytes initiate expression of myelin proteins critical for the achievement of proper functioning of the CNS [74]. Teneurin-4 (Tenm4) is a type II transmembrane protein that is highly expressed in the CNS and whose expression is induced in response to endoplasmic reticulum stress [75] and has been suggested to be involved in bipolar disorder in humans [76]. A mouse mutation, designated furue, which results in tremors and severe hypomyelination of small-diameter axons, reduces oligodendrocyte differentiation especially in the spinal cord of the CNS, and it has been associated with the absence of Tenm4 expression. Thus, Tenm4 is a critical regulator of oligodendrocyte differentiation and CNS myelination [55]. Herein we showed for the first time that in EAE mice the expression of Tenm4 is downregulated in the spinal cord and the treatment with EHP-101 reverses this downregulation probably as a result of the antiinflammatory activity of VCE-004.8.

In addition, oligodendrocytes are electrically and metabolically coupled through intercellular channels called gap junctions (GJs), composed of connexins Cx29, Cx32 and Cx47 [77], with other oligodendrocytes as well as with astrocytes. This glial network of communication plays an important role in the homeostasis of brain function [78, 79]. Several studies have also provided the role of oligodendrocyte connexins in acquired demyelinating CNS disorders, in particularMS and related 
experimental models $[80,81]$. They also appear to have a regulatory role in neuroinflammation as their absence further aggravates inflammatory demyelination [82]. Again, our results demonstrated that EHP101 prevented the downregulation of Gjc3 (connexin 29) expression in EAE mice vs control mice. In light of the relevance of Tenm4 and Gjc3 for oligodendrocyte function and myelin preservation, our results further support the potential of EHP-101 to be developed as a novel treatment of MS.

\section{Conclusions}

In conclusion, we have shown the protective effect of EHP-101 against demyelination and its capability to enhance remyelination. These results open new strategies for the treatment of MS and other demyelinating diseases, since novel therapies aimed at axonal remyelination are urgently needed.

\section{Abbreviations}

BBB: Blood-brain-barrier; CB2: Cannabinoid receptor type 2; CBD: Cannabidiol; Ccl2: C-C Motif Chemokine Ligand 2; Ccl4: C-C Motif Chemokine Ligand 4; CFA: Freund's adjuvant; CNS: Central nervous system; CPZ: Cuprizone; EAE: Experimental autoimmune encephalomyelitis; ECS: Endocannabinoid system; EPO: Erythropoietin; FGF-2: Fibroblast growth factor; GFAP: Glial fibrillary acidic protein; Gjc3: Gap junction gamma 3; Gjc3: Gap junction gamma 3; GO: Gene Ontology; GSTpi: Glutathione S-transferase pi; HIF: Hypoxia-inducible factor; Iba-1: Ionized calcium binding adaptor molecule 1; IGF-1: Insulin-like growth factor; II1b: Interleukin 1 beta; Il6: Interleukin 6; MBP: Myelin basic protein; MOG35-55: Myelin Oligodendrocyte Glycoprotein Peptide Fragment 35-55; MS: Multiple Sclerosis; Olig2: Oligodendrocyte transcription factor 2; OPC: Oligodendrocyte progenitor cell: Phosphate-buffered saline; PDGF-a: Plateletderived growth factor alpha; Peroxisome proliferator-activated receptor-gamma: PPAR囚; PHDs: Prolyl hydroxylases; RRMS: Relapsing-remitting MS; SMI-32: mouse anti-Neurofilament $\mathrm{H}$ (NF-H) Nonphosphorylated antibody; Tenm4: Teneurin 4; Timp1: Tissue inhibitor matrix metalloproteinase 1; TRP channels: Transient receptor potential channels; Vcam: Vascular cell adhesion molecule 1.

\section{Declarations}

Ethics approval and consent to participate: All experiments with laboratory animals were conducted according to European guidelines (directive 2010/63/EU), and the Ethics Committees on Animal Experimentation at the Cajal Institute (CSIC, Madrid) and the University of Córdoba (Córdoba, Spain) approved all the procedures described in this study (protocol numbers: 96 2013/03 CEEA-IC and 2018PI/02 (UCO).

Consent for publication: Not applicable.

Availability of data and material: Gene Expression Omnibus databank (accession no. GSE131854)

Competing interests: Carmen Navarrete is an employee of Emerald Health Pharmaceuticals. Adela GarcíaMartin is an employee of Emerald Health Biotechnology. Eduardo Muñoz is Chief Scientific Officer of 
Emerald Health Pharmaceuticals and a member of the Scientific Advisory Boards of Emerald Health Pharmaceuticals and Emerald Health Biotechnology.

Funding: This work was supported by grants SAF2017-87701-R (EM), SAF2016-76449-R (GC) from the Ministry of the Economy and Competition (MINECO) co-financed with the European Union FEDER funds. GC was also supported by the Red Española de Esclerosis Múltiple (REEM: RD16/0015/0021) sponsored by the Fondo de Investigación Sanitaria (FIS) (GC). This work was also partially supported by Emerald Health Pharmaceuticals (San Diego, USA).

Author contributions: $\mathrm{CN}, \mathrm{AGM}, \mathrm{LM}$, and AF performed in vivo experiments. GC and EM managed and designed the overall study. MGR and MAC performed the bioinformatic analysis. CN, AGM and MGR performed statistical analysis. $\mathrm{CN}$ and EM wrote the manuscript. All the authors approved the final manuscript.

Acknowledgements: We thank Carmen-Cabrero for revising the manuscript.

\section{References}

1. Lassmann $\mathrm{H}$ : Axonal and neuronal pathology in multiple sclerosis: what have we learnt from animal models. Exp Neurol 2010, 225:2-8.

2. Kipp M, Clarner T, Dang J, Copray S, Beyer C: The cuprizone animal model: new insights into an old story. Acta Neuropathol 2009, 118:723-736.

3. Scolding N, Franklin R: Axon loss in multiple sclerosis. Lancet 1998, 352:340-341.

4. Fox RJ, Cutter G, Chan A, Xiao J, Okwuokenye M, Levison D, Lewin J, Edwards MR, Marantz JL: Comparative Effectiveness Using A Matching-Adjusted Indirect Comparison Between DelayedRelease Dimethyl Fumarate and Fingolimod for The Treatment of Relapsing-Remitting Multiple Sclerosis. Value Health 2015, 18:A750.

5. Kister I, Chamot E, Salter AR, Cutter GR, Bacon TE, Herbert J: Disability in multiple sclerosis: a reference for patients and clinicians. Neurology 2013, 80:1018-1024.

6. Chiurchiu V, van der Stelt M, Centonze D, Maccarrone M: The endocannabinoid system and its therapeutic exploitation in multiple sclerosis: Clues for other neuroinflammatory diseases. Prog Neurobio/ 2018, 160:82-100.

7. llyasov AA, Milligan CE, Pharr EP, Howlett AC: The Endocannabinoid System and Oligodendrocytes in Health and Disease. Front Neurosci 2018, 12:733.

8. Feliu A, Bonilla Del Rio I, Carrillo-Salinas FJ, Hernandez-Torres G, Mestre L, Puente N, Ortega-Gutierrez S, Lopez-Rodriguez ML, Grandes P, Mecha M, Guaza C: 2-Arachidonoylglycerol Reduces Proteoglycans and Enhances Remyelination in a Progressive Model of Demyelination. $J$ Neurosci 2017, 37:8385-8398.

9. Bernal-Chico A, Canedo M, Manterola A, Victoria Sanchez-Gomez M, Perez-Samartin A, RodriguezPuertas R, Matute C, Mato S: Blockade of monoacylglycerol lipase inhibits oligodendrocyte 
excitotoxicity and prevents demyelination in vivo. Glia 2015, 63:163-176.

10. Barrie $\mathrm{N}$, Manolios $\mathrm{N}$ : The endocannabinoid system in pain and inflammation: Its relevance to rheumatic disease. Eur J Rheumatol 2017, 4:210-218.

11. Pistis M, O'Sullivan SE: The Role of Nuclear Hormone Receptors in Cannabinoid Function. Adv Pharmacol 2017, 80:291-328.

12. Pacher $P$, Mechoulam R: Is lipid signaling through cannabinoid 2 receptors part of a protective system? Prog Lipid Res 2011, 50:193-211.

13. Sanchez AJ, Garcia-Merino A: Neuroprotective agents: cannabinoids. Clin Immunol 2012, 142:57-67.

14. Pacher $P$, Kunos $G$ : Modulating the endocannabinoid system in human health and diseasesuccesses and failures. FEBS J 2013, 280:1918-1943.

15. Kong W, Li H, Tuma RF, Ganea D: Selective CB2 receptor activation ameliorates EAE by reducing Th17 differentiation and immune cell accumulation in the CNS. Cell Immunol 2014, 287:1-17.

16. Tomas-Roig J, Wirths O, Salinas-Riester G, Havemann-Reinecke U: The Cannabinoid CB1/CB2 Agonist WIN55212.2 Promotes Oligodendrocyte Differentiation In Vitro and Neuroprotection During the Cuprizone-Induced Central Nervous System Demyelination. CNS Neurosci Ther 2016, 22:387-395.

17. Navarrete C, Carrillo-Salinas F, Palomares B, Mecha M, Jimenez-Jimenez C, Mestre L, Feliu A, Bellido ML, Fiebich BL, Appendino G, et al: Hypoxia mimetic activity of VCE-004.8, a cannabidiol quinone derivative: implications for multiple sclerosis therapy. J Neuroinflammation 2018, 15:64.

18. Ricote M, Huang J, Fajas L, Li A, Welch J, Najib J, Witztum JL, Auwerx J, Palinski W, Glass CK: Expression of the peroxisome proliferator-activated receptor gamma (PPARgamma) in human atherosclerosis and regulation in macrophages by colony stimulating factors and oxidized low density lipoprotein. Proc Natl Acad Sci U S A 1998, 95:7614-7619.

19. Straus DS, Glass CK: Anti-inflammatory actions of PPAR ligands: new insights on cellular and molecular mechanisms. Trends Immunol 2007, 28:551-558.

20. Bernardo A, Minghetti L: PPAR-gamma agonists as regulators of microglial activation and brain inflammation. Curr Pharm Des 2006, 12:93-109.

21. Daynes RA, Jones DC: Emerging roles of PPARs in inflammation and immunity. Nat Rev Immunol 2002, 2:748-759.

22. Ricote M, Li AC, Willson TM, Kelly CJ, Glass CK: The peroxisome proliferator-activated receptorgamma is a negative regulator of macrophage activation. Nature 1998, 391:79-82.

23. Storer PD, Xu J, Chavis J, Drew PD: Peroxisome proliferator-activated receptor-gamma agonists inhibit the activation of microglia and astrocytes: implications for multiple sclerosis. $J$ Neuroimmunol 2005, 161:113-122.

24. Xu J, Racke MK, Drew PD: Peroxisome proliferator-activated receptor-alpha agonist fenofibrate regulates IL-12 family cytokine expression in the CNS: relevance to multiple sclerosis. $J$ Neurochem 2007, 103:1801-1810. 
25. Kanakasabai S, Pestereva E, Chearwae W, Gupta SK, Ansari S, Bright JJ: PPARgamma agonists promote oligodendrocyte differentiation of neural stem cells by modulating stemness and differentiation genes. PLoS One 2012, 7:e50500.

26. Bernardo A, Bianchi D, Magnaghi V, Minghetti L: Peroxisome proliferator-activated receptor-gamma agonists promote differentiation and antioxidant defenses of oligodendrocyte progenitor cells. $J$ Neuropathol Exp Neurol 2009, 68:797-808.

27. De Nuccio C, Bernardo A, De Simone R, Mancuso E, Magnaghi V, Visentin S, Minghetti L: Peroxisome proliferator-activated receptor gamma agonists accelerate oligodendrocyte maturation and influence mitochondrial functions and oscillatory Ca(2+) waves. J Neuropathol Exp Neurol 2011, 70:900-912.

28. Roth AD, Leisewitz AV, Jung JE, Cassina P, Barbeito L, Inestrosa NC, Bronfman M: PPAR gamma activators induce growth arrest and process extension in B12 oligodendrocyte-like cells and terminal differentiation of cultured oligodendrocytes. J Neurosci Res 2003, 72:425-435.

29. Inestrosa NC, Godoy JA, Quintanilla RA, Koenig CS, Bronfman M: Peroxisome proliferator-activated receptor gamma is expressed in hippocampal neurons and its activation prevents beta-amyloid neurodegeneration: role of Wnt signaling. Exp Cell Res 2005, 304:91-104.

30. Luna-Medina R, Cortes-Canteli M, Alonso M, Santos A, Martinez A, Perez-Castillo A: Regulation of inflammatory response in neural cells in vitro by thiadiazolidinones derivatives through peroxisome proliferator-activated receptor gamma activation. J Biol Chem 2005, 280:21453-21462.

31. Zhao X, Ou Z, Grotta JC, Waxham N, Aronowski J: Peroxisome-proliferator-activated receptor-gamma (PPARgamma) activation protects neurons from NMDA excitotoxicity. Brain Res 2006, 10731074:460-469.

32. Brodbeck J, Balestra ME, Saunders AM, Roses AD, Mahley RW, Huang Y: Rosiglitazone increases dendritic spine density and rescues spine loss caused by apolipoprotein E4 in primary cortical neurons. Proc Natl Acad Sci U S A 2008, 105:1343-1346.

33. McFarland HF, Martin R: Multiple sclerosis: a complicated picture of autoimmunity. Nat Immunol 2007, 8:913-919.

34. Stetler RA, Leak RK, Gan Y, Li P, Zhang F, Hu X, Jing Z, Chen J, Zigmond MJ, Gao Y: Preconditioning provides neuroprotection in models of CNS disease: paradigms and clinical significance. Prog Neurobiol 2014, 114:58-83.

35. Esen N, Serkin Z, Dore-Duffy P: Induction of vascular remodeling: a novel therapeutic approach in EAE. J Neurol Sci 2013, 333:88-92.

36. Girolamo F, Coppola C, Ribatti D, Trojano M: Angiogenesis in multiple sclerosis and experimental autoimmune encephalomyelitis. Acta Neuropathol Commun 2014, 2:84.

37. Yao SY, Soutto M, Sriram S: Preconditioning with cobalt chloride or desferrioxamine protects oligodendrocyte cell line (M03.13) from tumor necrosis factor-alpha-mediated cell death. J Neurosci Res 2008, 86:2403-2413.

38. Sattler MB, Merkler D, Maier K, Stadelmann C, Ehrenreich H, Bahr M, Diem R: Neuroprotective effects and intracellular signaling pathways of erythropoietin in a rat model of multiple sclerosis. Cell Death 
Differ 2004, 11 Suppl 2:S181-192.

39. Moransard M, Bednar M, Frei K, Gassmann M, Ogunshola OO: Erythropoietin reduces experimental autoimmune encephalomyelitis severity via neuroprotective mechanisms. J Neuroinflammation 2017, 14:202.

40. Garcia-Martin A, Garrido-Rodriguez M, Navarrete C, Caprioglio D, Palomares B, DeMesa J, Rollland A, Appendino G, Munoz E: Cannabinoid derivatives acting as dual PPARgamma/CB2 agonists as therapeutic agents for systemic sclerosis. Biochem Pharmacol 2019, 163:321-334.

41. Garcia-Martin A, Garrido-Rodriguez M, Navarrete C, Del Rio C, Bellido ML, Appendino G, Calzado MA, Munoz E: EHP-101, an oral formulation of the cannabidiol aminoquinone VCE-004.8, alleviates bleomycin-induced skin and lung fibrosis. Biochem Pharmacol 2018, 157:304-313.

42. Bolger AM, Lohse M, Usadel B: Trimmomatic: a flexible trimmer for Illumina sequence data. Bioinformatics 2014, 30:2114-2120.

43. Kim D, Langmead B, Salzberg SL: HISAT: a fast spliced aligner with low memory requirements. Nat Methods 2015, 12:357-360.

44. Liao Y, Smyth GK, Shi W: featureCounts: an efficient general purpose program for assigning sequence reads to genomic features. Bioinformatics 2014, 30:923-930.

45. Love MI, Huber W, Anders S: Moderated estimation of fold change and dispersion for RNA-seq data with DESeq2. Genome Bio/ 2014, 15:550.

46. Kuleshov MV, Jones MR, Rouillard AD, Fernandez NF, Duan Q, Wang Z, Koplev S, Jenkins SL, Jagodnik KM, Lachmann A, et al: Enrichr: a comprehensive gene set enrichment analysis web server 2016 update. Nucleic Acids Res 2016, 44:W90-97.

47. Yu G, Wang LG, Han Y, He QY: clusterProfiler: an R package for comparing biological themes among gene clusters. OMICS 2012, 16:284-287.

48. Klipper-Aurbach Y, Wasserman M, Braunspiegel-Weintrob N, Borstein D, Peleg S, Assa S, Karp M, Benjamini Y, Hochberg Y, Laron Z: Mathematical formulae for the prediction of the residual beta cell function during the first two years of disease in children and adolescents with insulin-dependent diabetes mellitus. Med Hypotheses 1995, 45:486-490.

49. Rasmussen S, Wang Y, Kivisakk P, Bronson RT, Meyer M, Imitola J, Khoury SJ: Persistent activation of microglia is associated with neuronal dysfunction of callosal projecting pathways and multiple sclerosis-like lesions in relapsing-remitting experimental autoimmune encephalomyelitis. Brain 2007, 130:2816-2829.

50. Kornek B, Lassmann H: Neuropathology of multiple sclerosis-new concepts. Brain Res Bull 2003, 61:321-326.

51. Storch MK, Bauer J, Linington C, Olsson T, Weissert R, Lassmann H: Cortical demyelination can be modeled in specific rat models of autoimmune encephalomyelitis and is major histocompatibility complex (MHC) haplotype-related. J Neuropathol Exp Neurol 2006, 65:1137-1142.

52. Bonzano L, Tacchino A, Roccatagliata L, Abbruzzese G, Mancardi GL, Bove M: Callosal contributions to simultaneous bimanual finger movements. J Neurosci 2008, 28:3227-3233. 
53. Manson SC, Palace J, Frank JA, Matthews PM: Loss of interhemispheric inhibition in patients with multiple sclerosis is related to corpus callosum atrophy. Exp Brain Res 2006, 174:728-733.

54. Manson SC, Wegner C, Filippi M, Barkhof F, Beckmann C, Ciccarelli O, De Stefano N, Enzinger C, Fazekas F, Agosta F, et al: Impairment of movement-associated brain deactivation in multiple sclerosis: further evidence for a functional pathology of interhemispheric neuronal inhibition. Exp Brain Res 2008, 187:25-31.

55. Suzuki N, Fukushi M, Kosaki K, Doyle AD, de Vega S, Yoshizaki K, Akazawa C, Arikawa-Hirasawa E, Yamada Y: Teneurin-4 is a novel regulator of oligodendrocyte differentiation and myelination of small-diameter axons in the CNS. J Neurosci 2012, 32:11586-11599.

56. Appendino G, Fontana G, Pollastro F: Comprehensive Natural Products II Chemistry and Biology Natural Products Drug Discovery 2010, 3:205-236.

57. del Rio C, Navarrete C, Collado JA, Bellido ML, Gomez-Canas M, Pazos MR, Fernandez-Ruiz J, Pollastro F, Appendino G, Calzado MA, et al: The cannabinoid quinol VCE-004.8 alleviates bleomycininduced scleroderma and exerts potent antifibrotic effects through peroxisome proliferator-activated receptor-gamma and CB2 pathways. Sci Rep 2016, 6:21703.

58. Lassmann H, Bradl M: Multiple sclerosis: experimental models and reality. Acta Neuropatho/2017, 133:223-244.

59. Brown DA, Sawchenko PE: Time course and distribution of inflammatory and neurodegenerative events suggest structural bases for the pathogenesis of experimental autoimmune encephalomyelitis. J Comp Neurol 2007, 502:236-260.

60. MacKenzie-Graham A, Rinek GA, Avedisian A, Gold SM, Frew AJ, Aguilar C, Lin DR, Umeda E, Voskuhl RR, Alger JR: Cortical atrophy in experimental autoimmune encephalomyelitis: in vivo imaging. Neuroimage 2012, 60:95-104.

61. Bennett J, Basivireddy J, Kollar A, Biron KE, Reickmann P, Jefferies WA, McQuaid S: Blood-brain barrier disruption and enhanced vascular permeability in the multiple sclerosis model EAE. $J$ Neuroimmunol 2010, 229:180-191.

62. Bakker DA, Ludwin SK: Blood-brain barrier permeability during Cuprizone-induced demyelination. Implications for the pathogenesis of immune-mediated demyelinating diseases. J Neurol Sci 1987, 78:125-137.

63. Zhang Z, Yan J, Chang Y, ShiDu Yan S, Shi H: Hypoxia inducible factor-1 as a target for neurodegenerative diseases. Curr Med Chem 2011, 18:4335-4343.

64. Jiang F, Frederick TJ, Wood TL: IGF-I synergizes with FGF-2 to stimulate oligodendrocyte progenitor entry into the cell cycle. Dev Biol 2001, 232:414-423.

65. Schito L, Rey S, Tafani M, Zhang H, Wong CC, Russo A, Russo MA, Semenza GL: Hypoxia-inducible factor 1-dependent expression of platelet-derived growth factor B promotes lymphatic metastasis of hypoxic breast cancer cells. Proc Natl Acad Sci U S A 2012, 109:E2707-2716.

66. Calvani M, Rapisarda A, Uranchimeg B, Shoemaker RH, Melillo G: Hypoxic induction of an HIF1alpha-dependent bFGF autocrine loop drives angiogenesis in human endothelial cells. Blood 2006, 
107:2705-2712.

67. Trapp BD, Peterson J, Ransohoff RM, Rudick R, Mork S, Bo L: Axonal transection in the lesions of multiple sclerosis. N Engl J Med 1998, 338:278-285.

68. Ferguson B, Matyszak MK, Esiri MM, Perry VH: Axonal damage in acute multiple sclerosis lesions. Brain 1997, 120 (Pt 3):393-399.

69. Popescu BF, Lucchinetti CF: Pathology of demyelinating diseases. Annu Rev Pathol 2012, 7:185-217.

70. Song GJ, Suk K: Pharmacological Modulation of Functional Phenotypes of Microglia in Neurodegenerative Diseases. Front Aging Neurosci 2017, 9:139.

71. Peterson JW, Bo L, Mork S, Chang A, Trapp BD: Transected neurites, apoptotic neurons, and reduced inflammation in cortical multiple sclerosis lesions. Ann Neuro/ 2001, 50:389-400.

72. Werner P, Pitt D, Raine CS: Multiple sclerosis: altered glutamate homeostasis in lesions correlates with oligodendrocyte and axonal damage. Ann Neurol 2001, 50:169-180.

73. Tsai HH, Macklin WB, Miller RH: Distinct modes of migration position oligodendrocyte precursors for localized cell division in the developing spinal cord. J Neurosci Res 2009, 87:3320-3330.

74. Louis JC, Magal E, Muir D, Manthorpe M, Varon S: CG-4, a new bipotential glial cell line from rat brain, is capable of differentiating in vitro into either mature oligodendrocytes or type-2 astrocytes. $J$ Neurosci Res 1992, 31:193-204.

75. Wang XZ, Kuroda M, Sok J, Batchvarova N, Kimmel R, Chung P, Zinszner H, Ron D: Identification of novel stress-induced genes downstream of chop. EMBO J 1998, 17:3619-3630.

76. Psychiatric GCBDWG: Large-scale genome-wide association analysis of bipolar disorder identifies a new susceptibility locus near ODZ4. Nat Genet 2011, 43:977-983.

77. Kleopa KA, Orthmann JL, Enriquez A, Paul DL, Scherer SS: Unique distributions of the gap junction proteins connexin29, connexin32, and connexin47 in oligodendrocytes. Glia 2004, 47:346-357.

78. Orthmann-Murphy JL, Abrams CK, Scherer SS: Gap junctions couple astrocytes and oligodendrocytes. J Mol Neurosci 2008, 35:101-116.

79. Giaume C, Koulakoff A, Roux L, Holcman D, Rouach N: Astroglial networks: a step further in neuroglial and gliovascular interactions. Nat Rev Neurosci 2010, 11:87-99.

80. Markoullis K, Sargiannidou I, Gardner C, Hadjisavvas A, Reynolds R, Kleopa KA: Disruption of oligodendrocyte gap junctions in experimental autoimmune encephalomyelitis. Glia 2012, 60:10531066.

81. Markoullis K, Sargiannidou I, Schiza N, Hadjisavvas A, Roncaroli F, Reynolds R, Kleopa KA: Gap junction pathology in multiple sclerosis lesions and normal-appearing white matter. Acta Neuropathol 2012, 123:873-886.

82. Papaneophytou CP, Georgiou E, Karaiskos C, Sargiannidou I, Markoullis K, Freidin MM, Abrams CK, Kleopa KA: Regulatory role of oligodendrocyte gap junctions in inflammatory demyelination. Glia 2018, 66:2589-2603. 


\section{Tables}

Table 1. Primers used in real-time PCR analysis.

\begin{tabular}{|ccc|}
\hline Genes & Forward $\left(\mathbf{5}^{\prime}-\mathbf{3}\right.$ ) & Reverse $\left(\mathbf{5}^{\prime}-\mathbf{3} \mathbf{3}^{\prime}\right)$ \\
\hline IL-6 & GTATGAACAACGATGATGCACTTG & ATGGTACTCCAGAAGACCAGAGGA3 \\
\hline IL-1b & CTCCACCTCAATGGACAGAA & GCCGTCTTTCATTACACAGG \\
\hline CCL2 & GGGCCTGCTGTTCACAGTT & CCAGCCTACTCATTGGGAT \\
\hline Gjc3 & AGAAACAGCAGGAAGTGGGA & AACACCATGAAGCTCTGCGT \\
\hline TIMP1 & TCCTCTTGTTGCTATCACTGATAGCTT & CGCTGGTATAAGGTGGTCTCGTT \\
\hline Tenm4 & GTGGACAAGTTTGGGCTCATTTAT & GGGTTGATGGCTAAGTCTGTGG \\
\hline VCAM & AGTTGGGGATTCGGTTGTTC & CATTCCTTACCACCCCATTG \\
\hline GAPDH & TGGCAAAGTGGAGATTGTTGCC & AAGATGGTGATGGGCTTCCCG \\
\hline
\end{tabular}

\section{Figures}


a

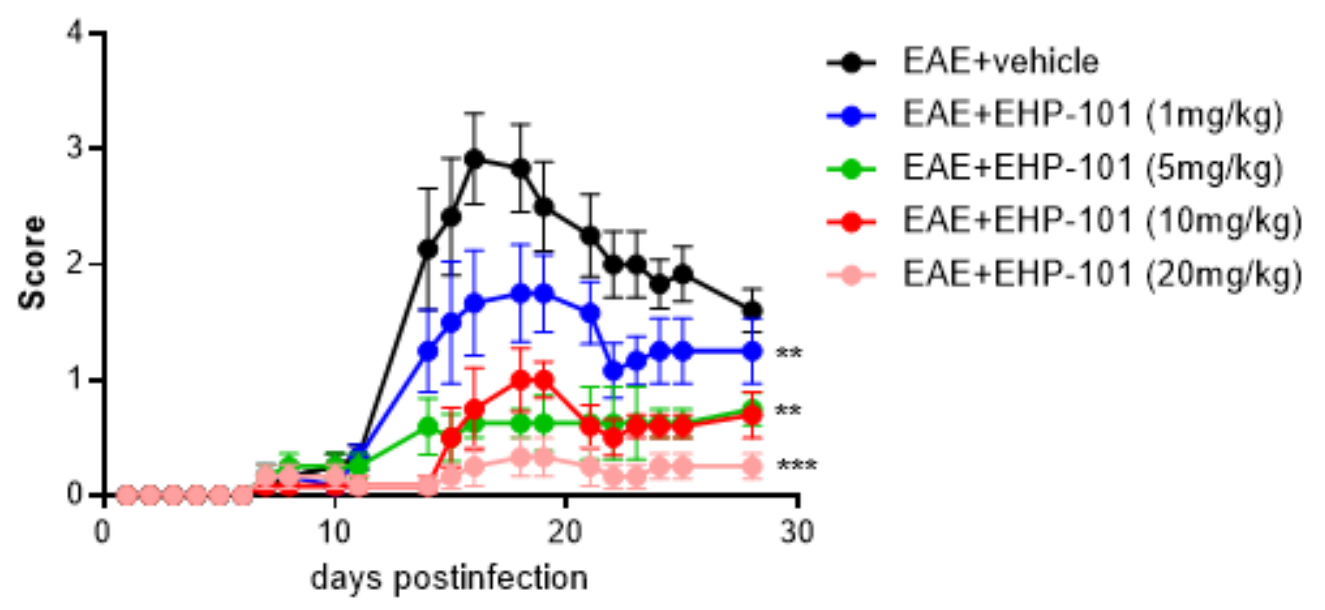

b

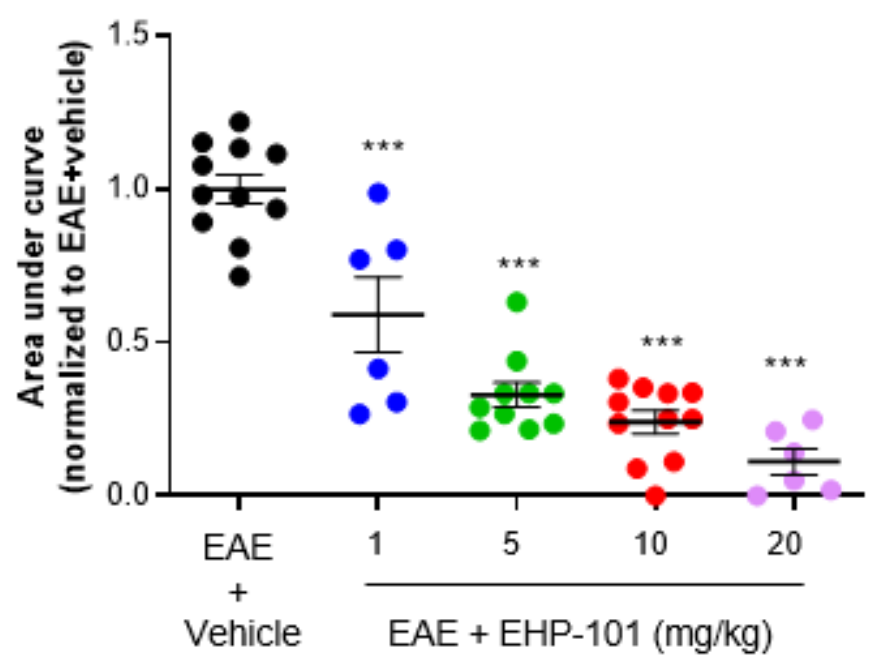

Figure 1

EHP-101 attenuates clinical severity and neuropathology in EAE model. (a) EHP-101 significantly ameliorated the clinical signs and progression of EAE. Results are expressed as mean \pm SEM $(n=6$ animals per group). ${ }^{*} \mathrm{p}<0.01,{ }^{* \star *} \mathrm{p}<0.001 \mathrm{EAE}+\mathrm{EHP}-101 \mathrm{vs} \mathrm{EAE}+$ Vehicle (one-way ANOVA followed by the Tukey's post-hoc test). (b) Clinical activity was quantified by measuring the area under curve. Results are expressed as mean \pm SEM ( $n=6$ to 11 animals per group). ${ }^{\star \star *} p<0.001$ EAE + EHP-101 vs $\mathrm{EAE}+$ Vehicle (one-way ANOVA followed by the Tukey's post-hoc test). 
a

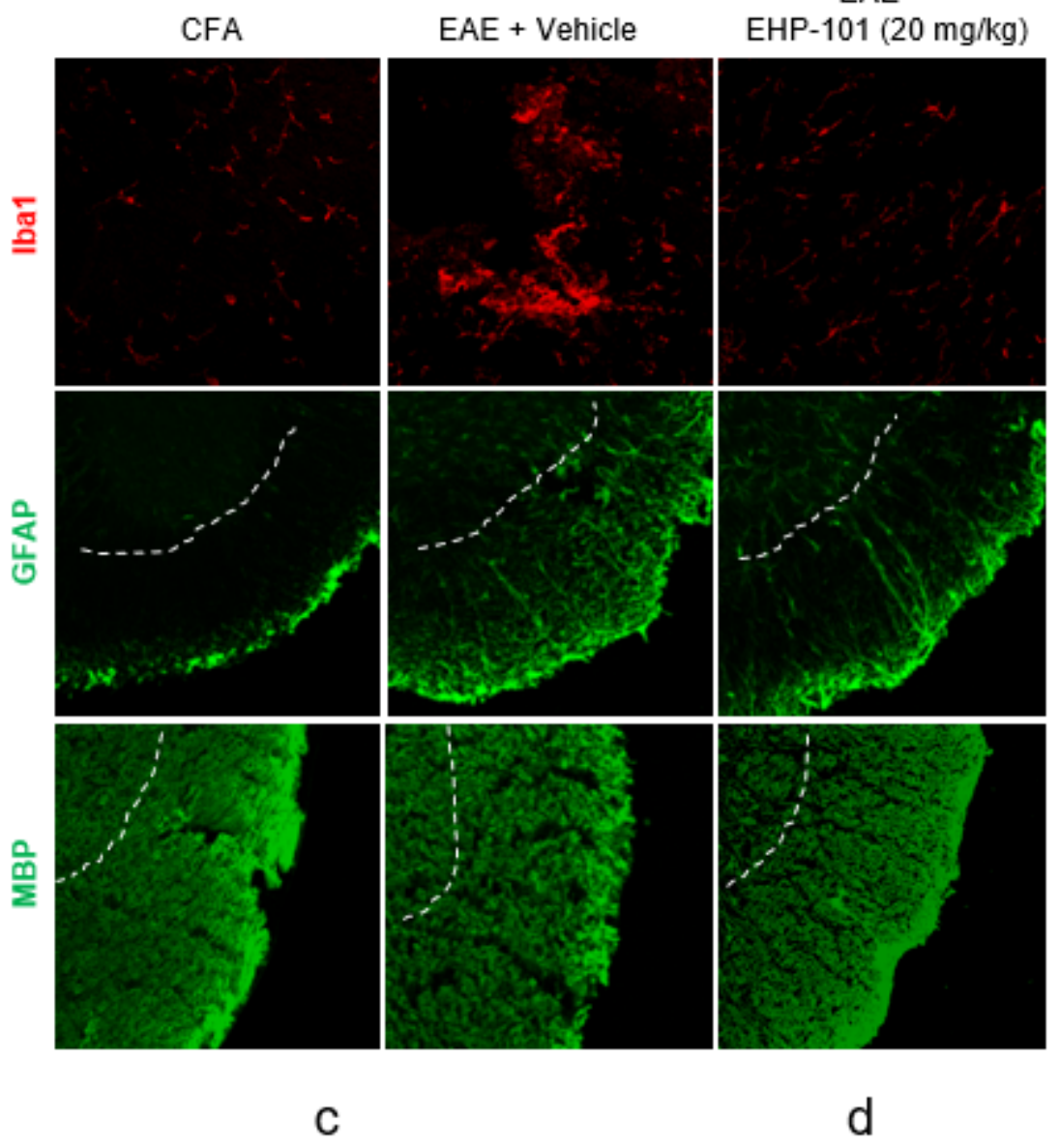

b

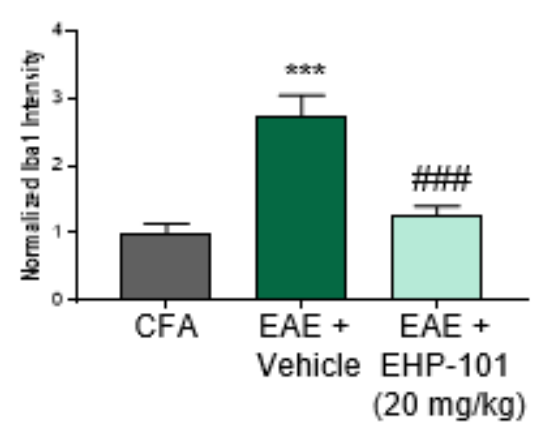

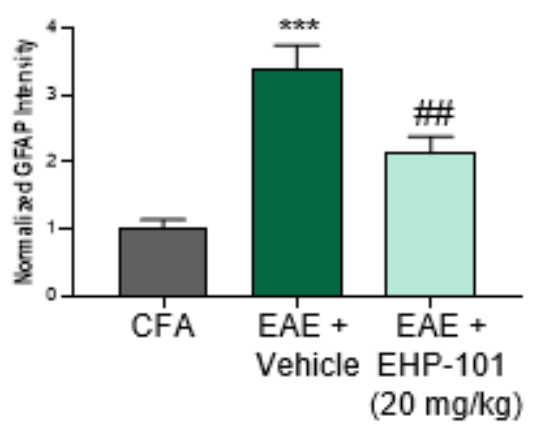

$\mathrm{EAE}+$

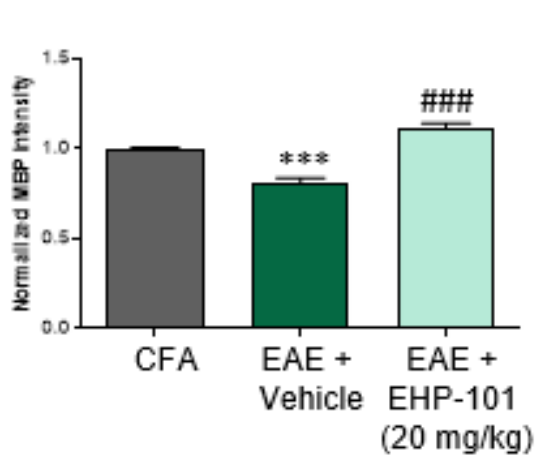

\section{Figure 2}

EHP-101 significantly reduces glial reactivity and preserves myelin structure in the spinal cord of EAE animals. (a) Cross-sectional images of thoracic spinal cord cross-sections of $50 \mu \mathrm{m}$ thick, in which immunofluorescence with anti-lba1, GFAP and myelin staining MBP were performed. The quantifications of each marker $(b-d)$ are shown as mean \pm SEM, and significance was determined by one-way ANOVA followed by the Tukey's post-hoc test. ${ }^{* \star} p<0.001 \mathrm{EAE}+$ Vehicle vs CFA; \#\#p $<0.01, \# \# \# p<0.001 \mathrm{EAE}+$ EHP-101 vs EAE + Vehicle. 
a

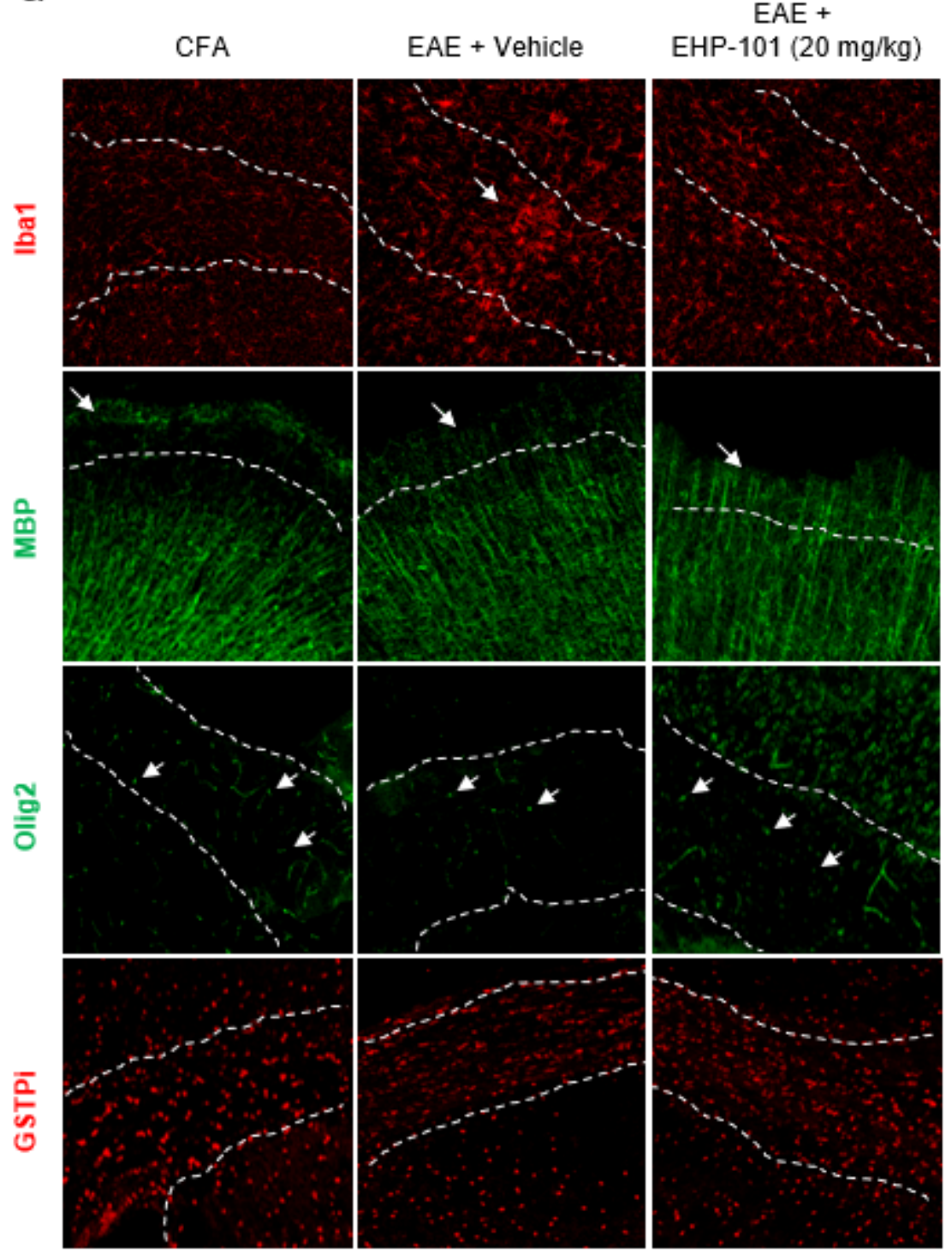

b
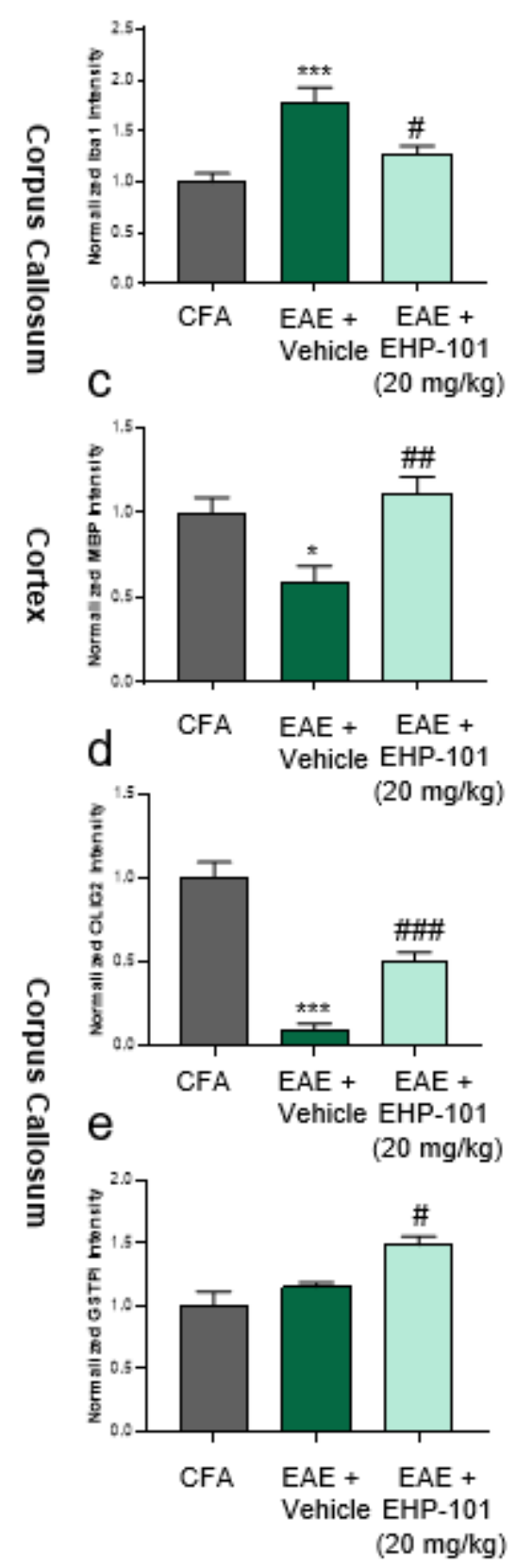

\section{Figure 3}

Demyelination with persistent activation of microglia and loss of Olig2 expression was prevented by EHP101 treatment. (a) Representative confocal microscopy images of immunolabeled cells for Iba1, MBP, Olig2 and GSTpi expression in corpus callosum and cortex. (b-e) The quantifications of each marker are shown as mean \pm SEM, and significance was determined by one-way ANOVA followed by the Tukey's post-hoc test. ${ }^{*} p<0.05,{ }^{\star \star *} p<0.001 \mathrm{EAE}+$ Vehicle vs CFA; \#p $<0.05, \# \# p<0.01, \# \# \# p<0.001 \mathrm{EAE}+$ EHP-101 vs EAE + Vehicle. 
a

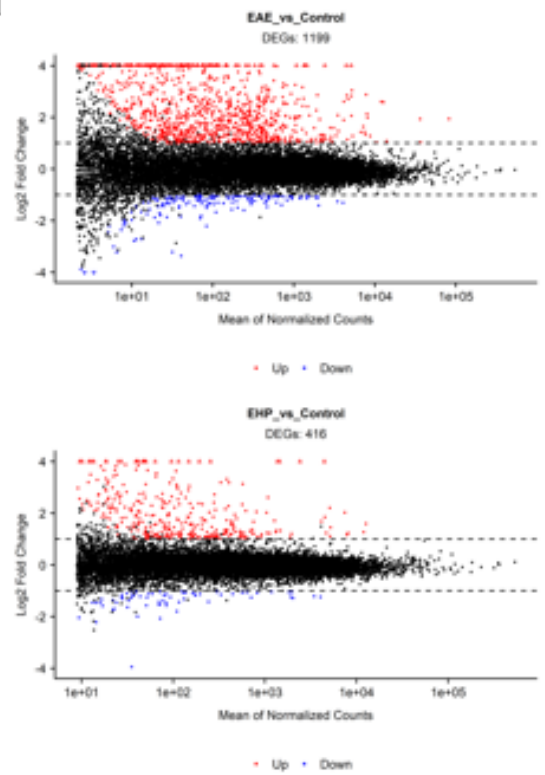

C

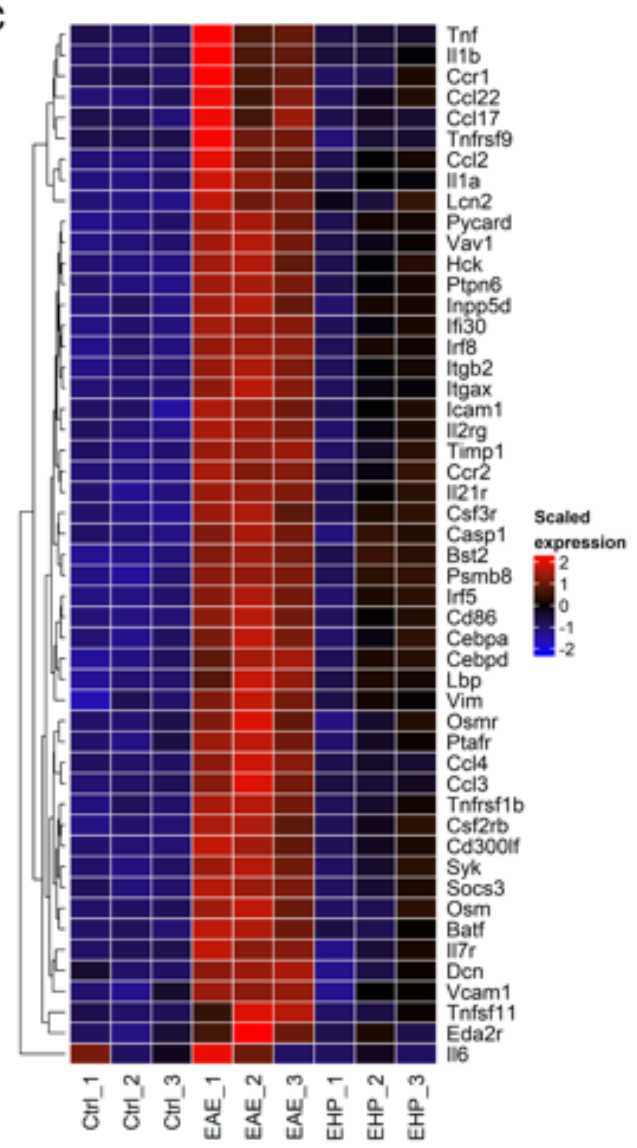

b

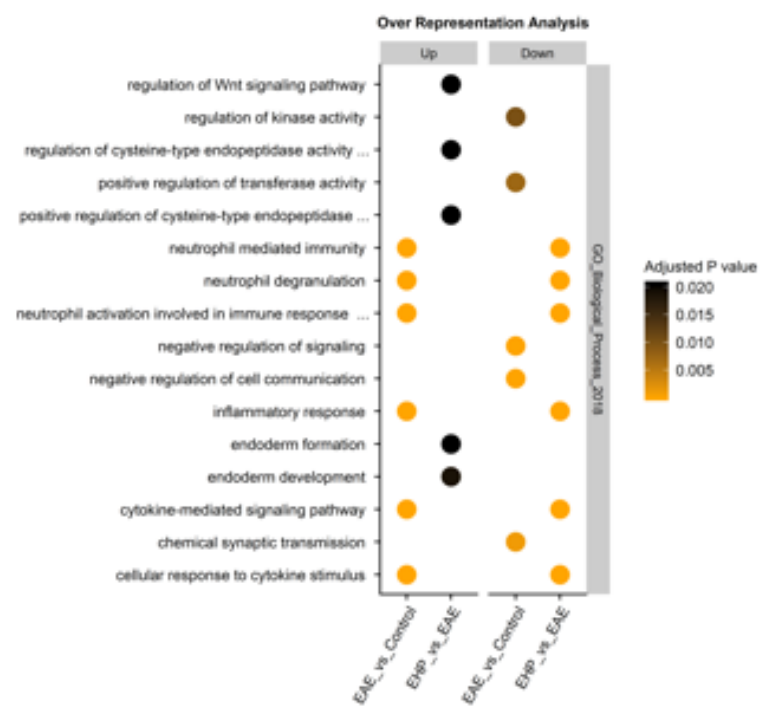

d
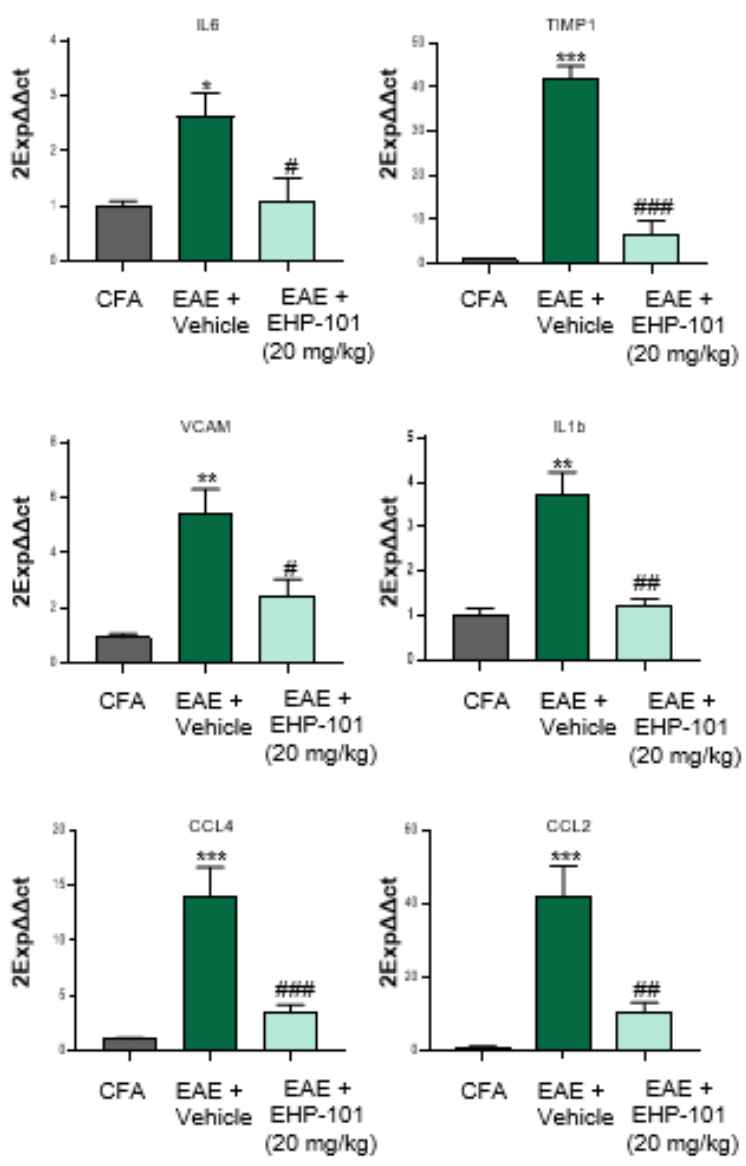

\section{Figure 4}

Gene expression profiling of the effect of EHP-101 in EAE model. (a) MA plots of the EAE or EAE+EHP-101 vs control comparisons. The $X$ axis represents the average expression as the mean of normalized counts while the $Y$ axis indicates the magnitude of the change as the log 2 transformed fold change. The color indicates genes that surpassed the cutoff of adjusted $P<0.05$ and fold change $<-2$ (blue) or $>2$ (red). (b) Functional analysis results for genes that surpassed the cutoff in EAE vs Control and EAE+EHP-101 vs 
EAE comparisons. Dots indicate a significant over-representation (adjusted $\mathrm{P}<0.05$ ) of a Gene Ontology (Biological Process) term ( $Y$ axis) in a set of up or down regulated genes (X-axis). (c) Heatmap depicting the expression levels for selected genes included in the "cytokine-mediated signaling pathway". (d) The mRNA expression for inflammatory marker in spinal cord (IL-6, Timp1, VCAM, IL 1b, CCL2 and CCL4) was quantified by qPCR and normalized versus GAPDH. Data represent the mean \pm SEM, and significance was determined by one-way ANOVA followed by the Tukey's post-hoc test. ${ }^{*} p<0.05,{ }^{*} p<0.01,{ }^{* \star} p<$ $0.001 \mathrm{EAE}+$ Vehicle vs CFA; $\# p<0.05, \# \# p<0.01, \# \# \# p<0.001 \mathrm{EAE}+\mathrm{EHP}-101 \mathrm{vs} \mathrm{EAE}+$ Vehicle.

a
Down
Up

EAE/Control

b

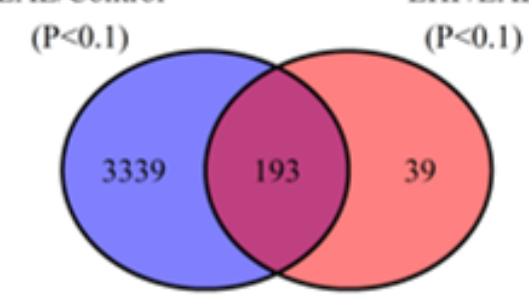

C
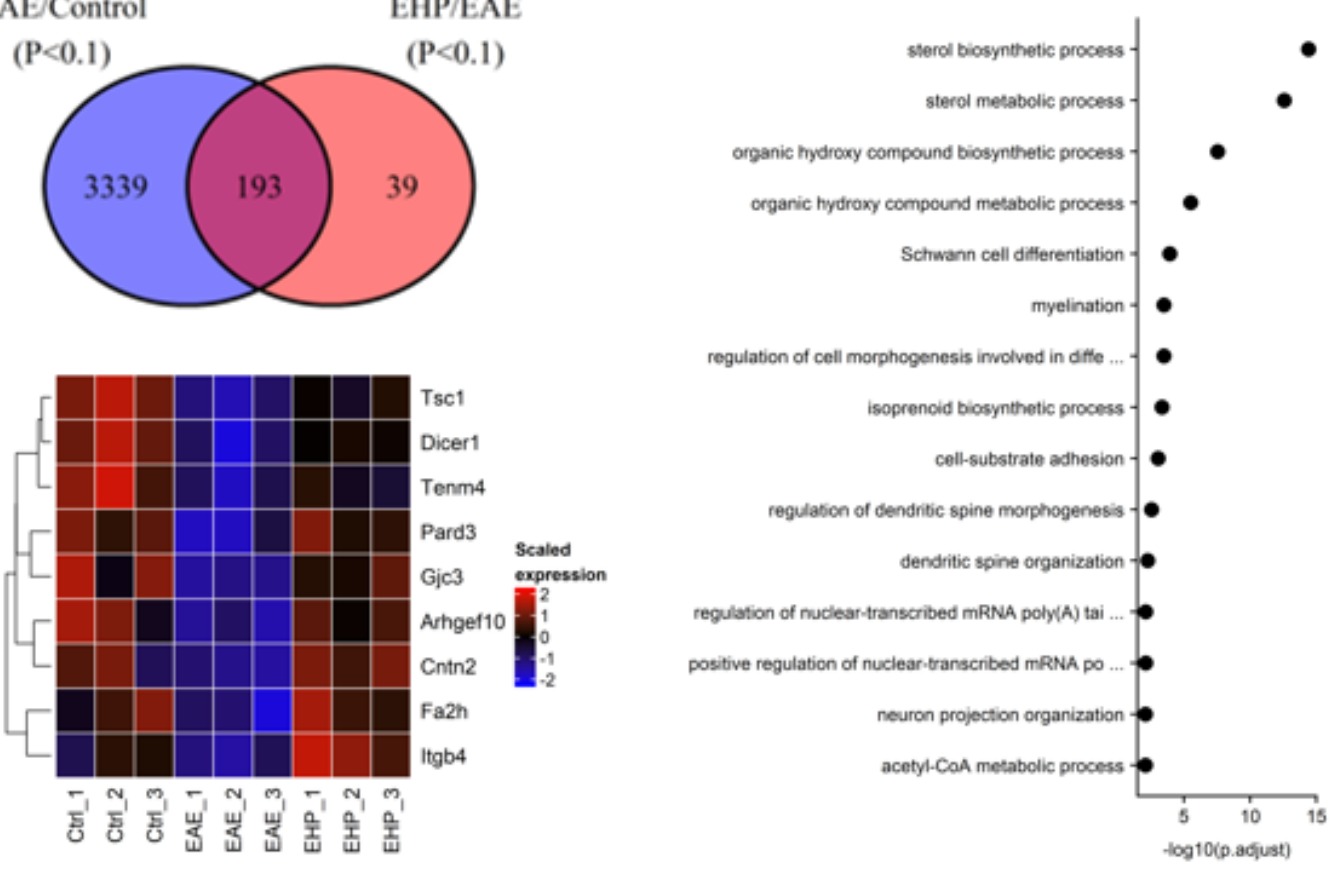

d

e
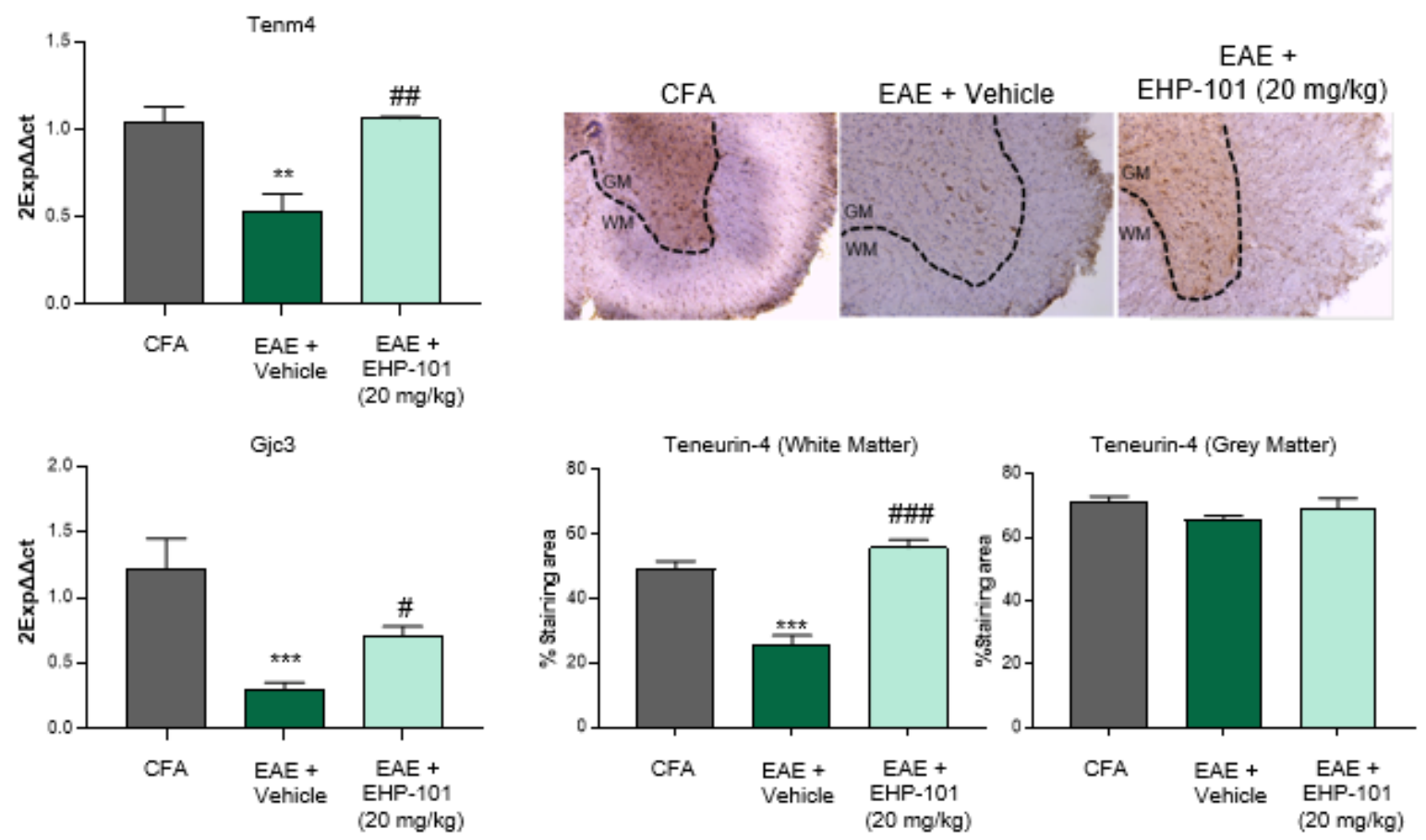


\section{Figure 5}

EHP-101 treatment normalized the expression of genes associated with oligodendrocyte function. (a) Venn Diagram indicating the overlap between the sets of down regulated genes at EAE vs Control comparison (Adjusted $\mathrm{P}<0.1$ and fold change $<0$ ) and up regulated genes at EAE+EHP-101 vs EAE comparison (Adjusted $\mathrm{P}<0.1$ and fold change $>0$ ). (b) Functional analysis results for the set of 193 overlapping genes. The scatter plot represents the significance of the enrichment for the top 15 overrepresented Gene Ontology (Biological Process) terms as the -log10 transformed adjusted P value. (c) Heatmap depicting the expression levels for genes annotated with the "myelination" GO term included in the set of 193 overlapping features. (d) The mRNA expression for myelination-related genes (Tenm4 and Gjc3) was quantified by qPCR and normalized versus GAPDH. (e) Immunohistochemistry labelling of spinal cord for Teneurin-4 in CFA, EAE + + Vehicle and EAE + EHP-101 are shown. The quantification of expression of Teneurin- 4 in the white and the grey matter (bottom panel). Data represent the mean \pm SEM and significance were determined by one-way ANOVA followed by the Tukey's post-hoc test. ${ }^{*} \mathrm{p}<0.01$, $\star \star \star p<0.001 \mathrm{EAE}+$ Vehicle vs CFA; \#p $<0.05, \# \# p<0.01, \# \# \# p<0.001 \mathrm{EAE}+\mathrm{EHP}-101 \mathrm{vs} \mathrm{EAE}+$ Vehicle. 
a

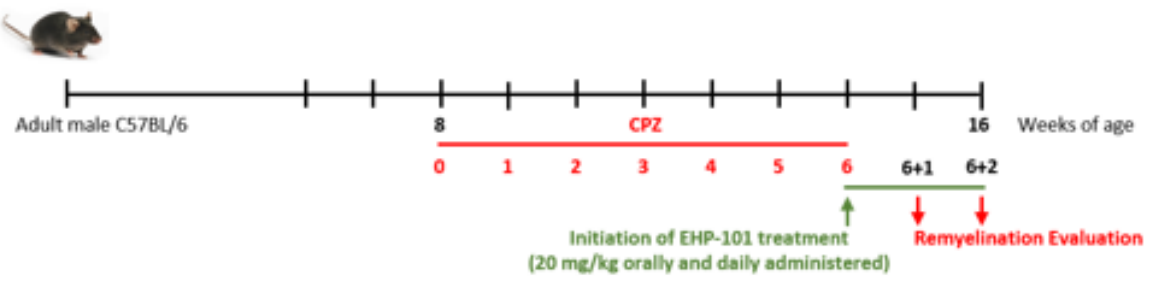

b

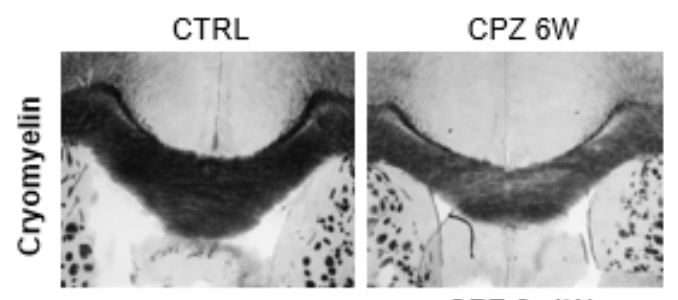

$\mathrm{CPZ} 6+1 \mathrm{~W}+$

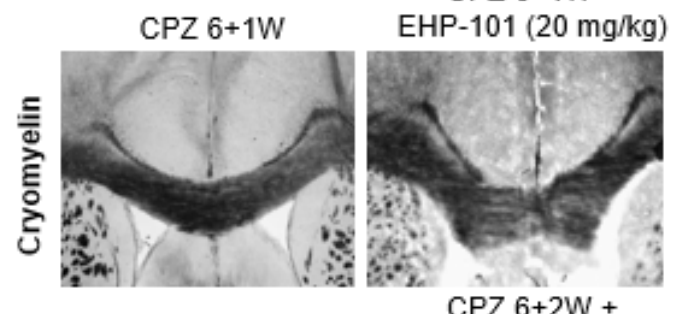

$\mathrm{CPZ} 6+2 \mathrm{~W}+$

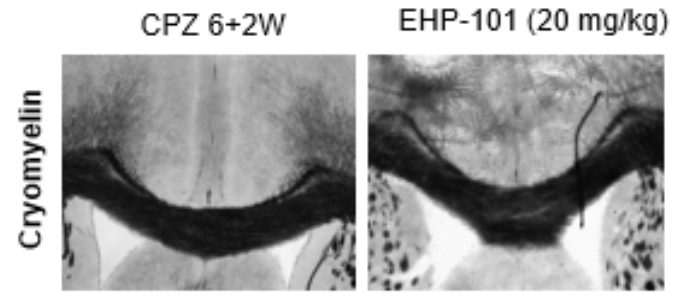

C
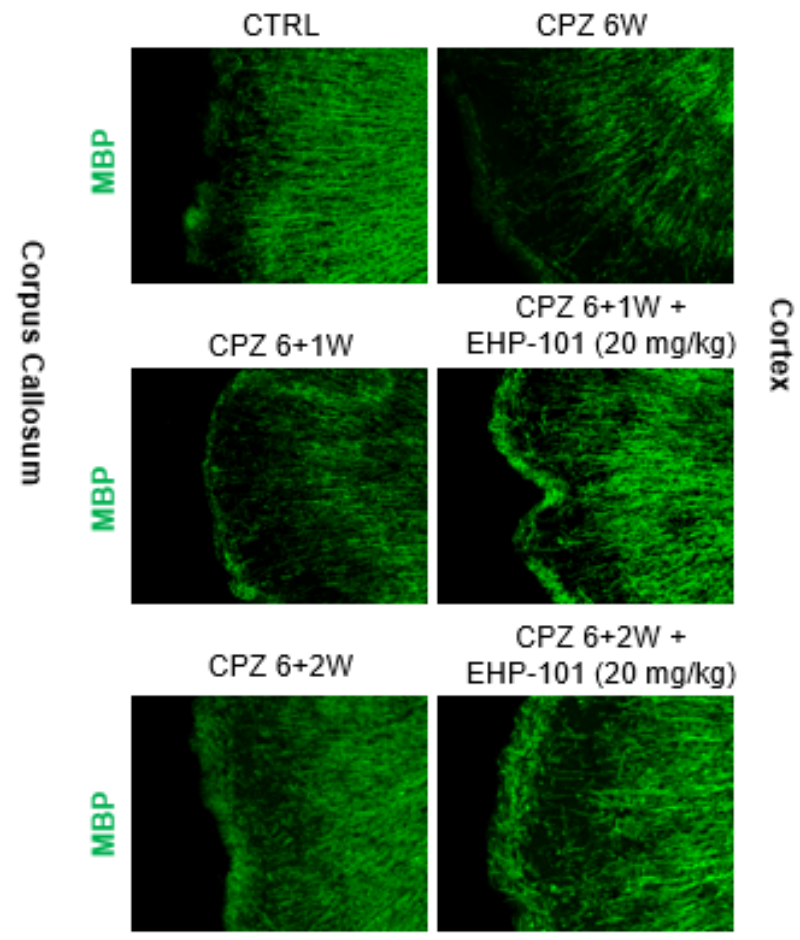

d

e
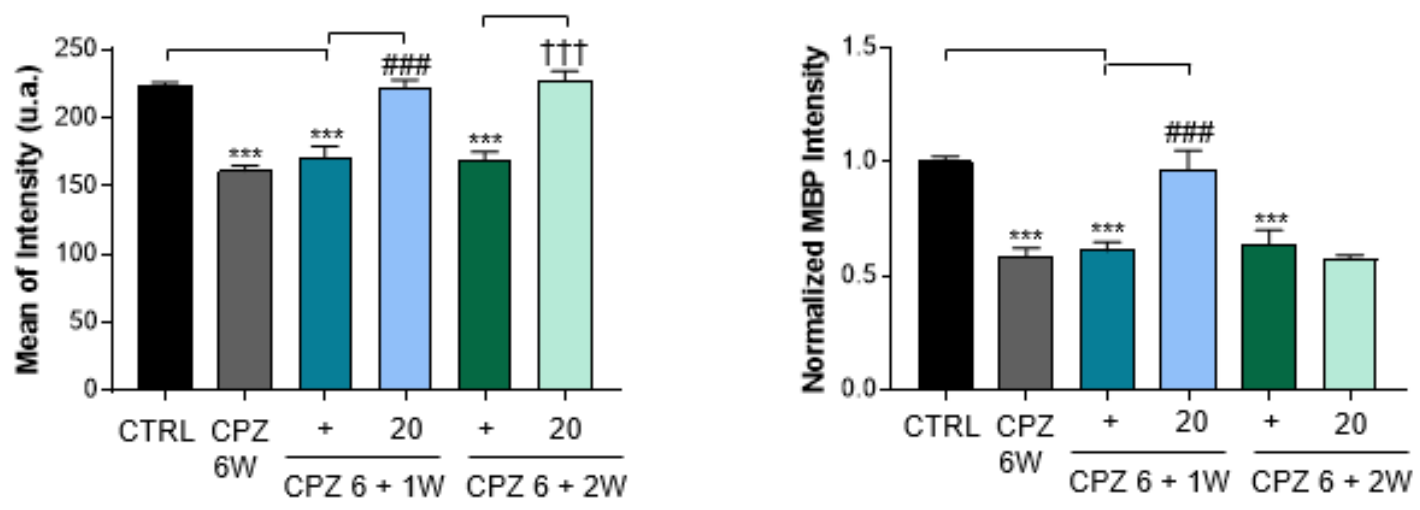

\section{Figure 6}

Effect of EHP-101 treatment on remyelination in acute CPZ-induced demyelination. (a) Experimental procedure (b). Histological study of myelin by Cryomyelin staining in corpus callosum and quantification of the mean intensity is shown (d) ( $n=5$ animals per group). (c) Immunodetection of MBP in the cerebral cortex, and (e) the quantification of MBP immunoreactivity. Data represent the mean \pm SEM, and significance was determined by one-way ANOVA followed by the Tukey's post-hoc test. ${ }^{* \star *}$ p $<0.001 \mathrm{CPZ}$ 
6W or CPZ 6W + 1 or CPZ 6W + 2 vs Control; \#\#\#p < $0.001 \mathrm{CPZ} \mathrm{6W} \mathrm{+1} \mathrm{+} \mathrm{EHP-101} \mathrm{vs} \mathrm{CPZ} \mathrm{6W} \mathrm{+1,} \mathrm{†十+p} \mathrm{<}$ $0.001 \mathrm{CPZ} 6 \mathrm{~W}+2+\mathrm{EHP}-101 \mathrm{vs} \mathrm{CPZ} 6 \mathrm{~W}+2$.

a
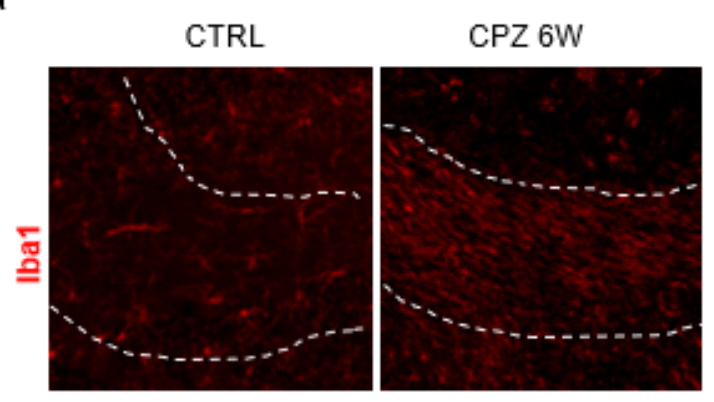

b
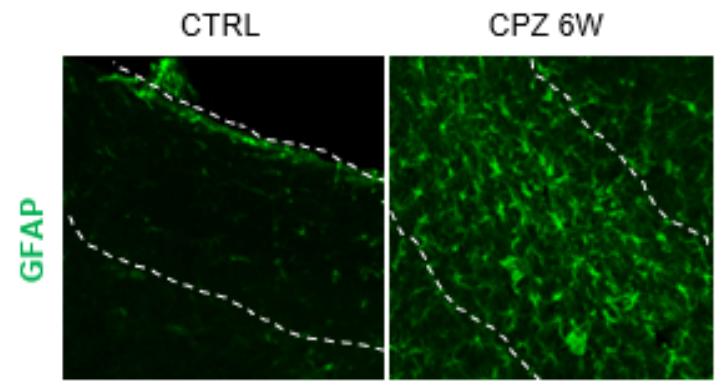

CPZ 6+1W +

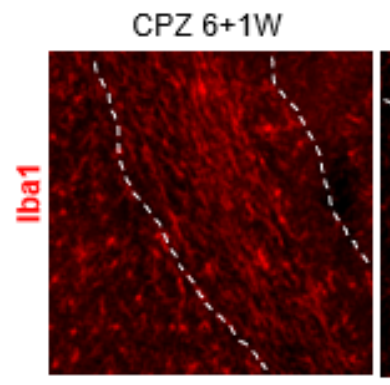

EHP-101 $(20 \mathrm{mg} / \mathrm{kg})$

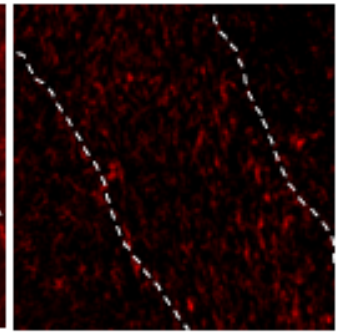

CPZ 6+2W +

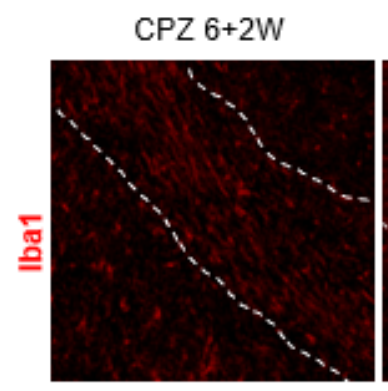

EHP-101 $(20 \mathrm{mg} / \mathrm{kg})$

C
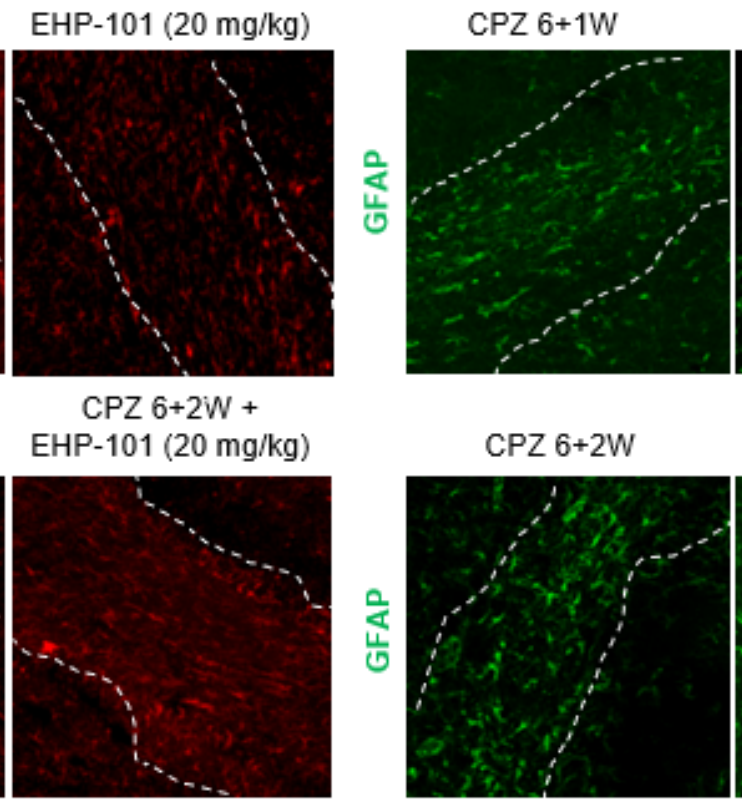

CPZ 6+1W +

EHP-101 $(20 \mathrm{mg} / \mathrm{kg})$

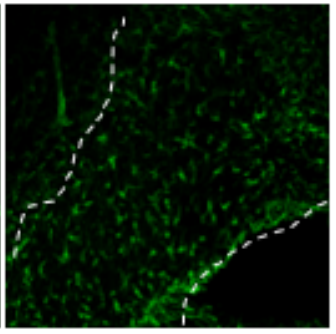

CPZ 6+2W +

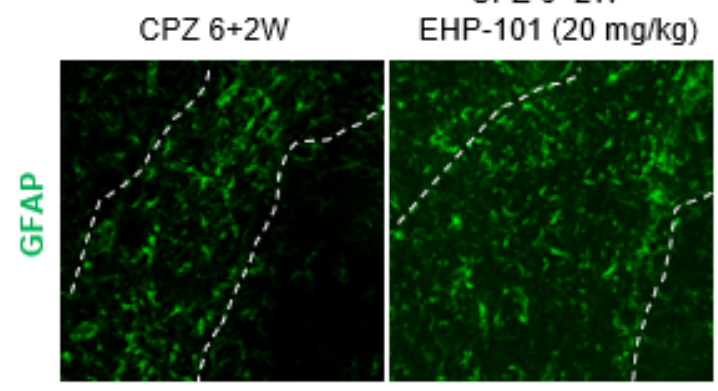

$\mathrm{d}$
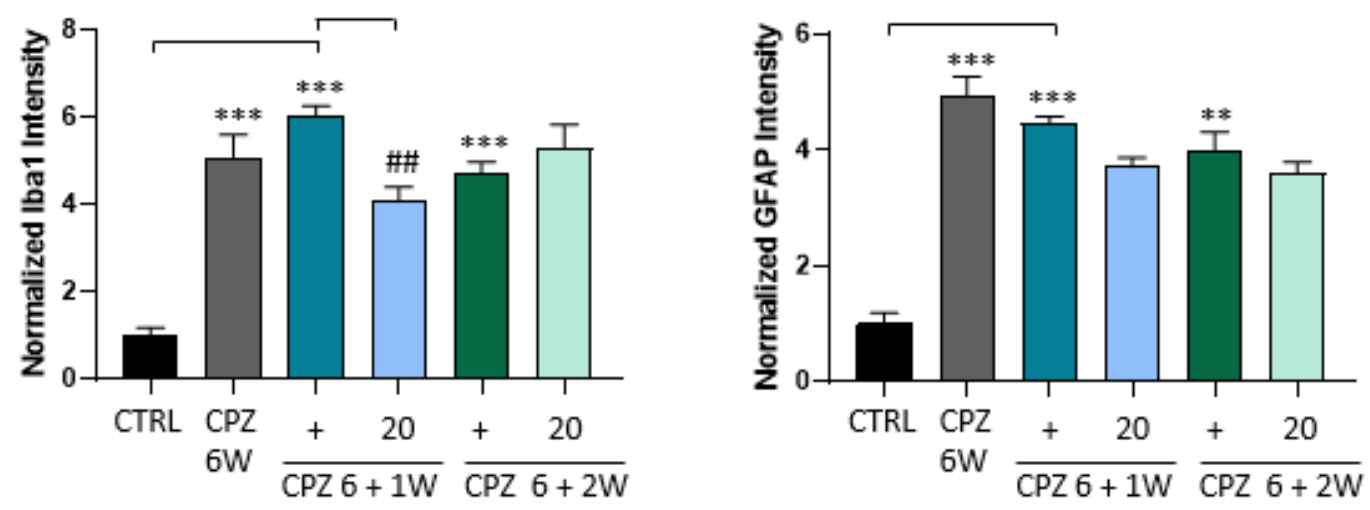

Figure 7

Impact of EHP-101 treatment on microglia and astrocytes activation in acute CPZ-induced demyelination. (a) Expression of Iba1 in corpus callosum and quantification (c). (b) Astrogliosis was determined by immunofluorescence studies of GFAP in corpus callosum and quantification of intensity is shown (d). 
Data represent the mean \pm SEM, and significance was determined by one-way ANOVA followed by the

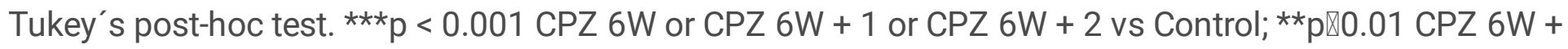
2 vs Control; \#\#p < $0.01 \mathrm{CPZ} 6 \mathrm{~W}+1+\mathrm{EHP}-101$ vs CPZ 6W +1.

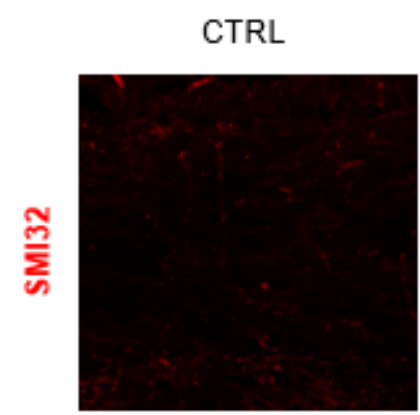

CPZ $6+1 W$

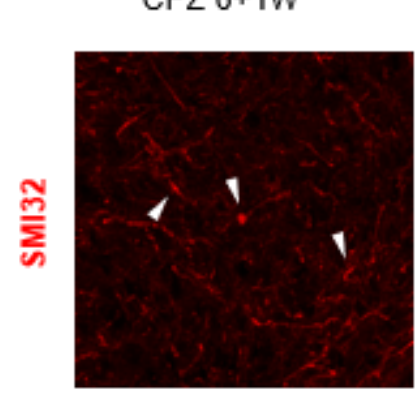

CPZ $6+2 W$
CPZ 6W

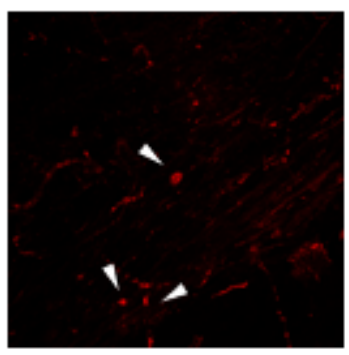

$\mathrm{CPZ} 6+1 \mathrm{~W}+$

EHP-101 $(20 \mathrm{mg} / \mathrm{kg})$

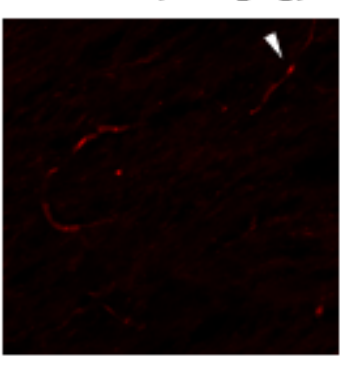

CPZ 6+2W +

EHP-101 $(20 \mathrm{mg} / \mathrm{kg})$
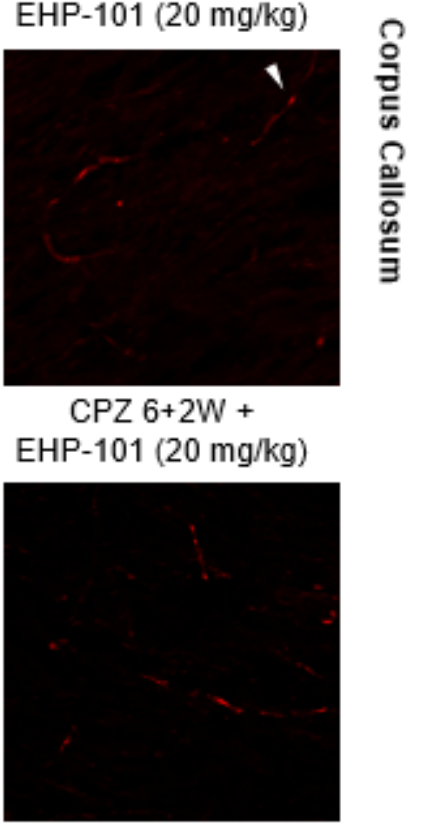

\section{Figure 8}

EHP-101 reduces axonal degeneration induced by CPZ. Representative images of immunostaining of SMI-32+ cells in the corpus callosum of different groups of animals. 